\title{
The Remarkable Eclipsing Asynchronous AM Herculis Binary RX J19402-1025
}

\author{
JOSEPH PATTERSON ${ }^{1}$ \\ Department of Astronomy, Columbia University, 538 W. 120th Street, New York, New York 10027 \\ Electronic mail: jop@carmen.phys.columbia.edu \\ DAVID R. SKILLMAN \\ Center for Basement Astrophysics (East), 9517 Washington Avenue, Laurel, Maryland 20723
}

\section{JOHN THORSTENSEN}

Department of Physics and Astronomy, 6127 Wilder Laboratory, Dartmouth College, Hanover, New Hampshire 03755 Electronic mail: thorstensen@dartmouth.edu

\author{
COEL HELlier ${ }^{2}$ \\ Department of Physics, Keele University, Keele, Staffordshire ST5 5BG, United Kingdom \\ Electronic mail: ch@keele.astro.ac.uk \\ Received 1994 December 8; accepted 1995 January 11
}

\begin{abstract}
We report on two years of photometric and spectroscopic observation of the recently discovered AM Herculis star RX $\mathrm{J} 19402-1025$. A sharp eclipse feature is present in the optical and X-ray light curves, repeating with a period of $12116.290 \pm 0.003 \mathrm{~s}$. The out-of-eclipse optical waveform contains approximately equal contributions from a signal at the same period and another signal at $12150 \mathrm{~s}$. As these signals drift in and out of phase, the wave form of the light curve changes in a complex but predictable manner. After one entire "supercycle" of 50 days (the beat period between the shorter periods), the light curve returns to its initial shape. We present long-term ephemerides for each of these periods. It is highly probable that the eclipse period is the underlying orbital period, while the magnetic white dwarf rotates with $P=12150 \mathrm{~s}$. The eclipses appear to be eclipses of the white dwarf by the secondary star. But there is probably also a small obscuring effect from cold gas surrounding the secondary, especially on the orbit-leading side where the stream begins to fall towards the white dwarf. The latter hypothesis can account for several puzzling effects in this star, as well as the tendency among most AM Her stars for the sharp emission-line components to slightly precede the actual motion of the secondary. The presence of eclipses in an asynchronous AM Her star provides a marvelous opportunity to study how changes in the orientation of magnetic field lines affect the accretion flows. Repeated polarimetric light curves and high-resolution studies of the emission lines are now critical to exploit this potential.
\end{abstract}

\section{INTRODUCTION}

For the past 15 years, one of the most intriguing objects in the X-ray sky has been the source identified with the Seyfert galaxy NGC 6814. This is because the X-ray flux shows very rapid variations on time scales of a few hundred seconds (Tennant et al. 1981; Tennant and Mushotzky 1983), and a strict periodicity of $P=3.4 \mathrm{hr}$ which manifests itself both in the sharp dips and in a smooth variation around the cycle (Mittaz and Branduardi-Raymond 1989; Done et al. 1992;

\footnotetext{
${ }^{1}$ Visiting Astronomer, Cerro Tololo Inter-American Observatory, which is operated by the Associated Universities for Research in Astronomy, Inc. (AURA), under contract with the National Science Foundation.

${ }^{2}$ Also at Department of Astronomy and McDonald Observatory, University of Texas at Austin, Austin, TX 78712.
}

Leighly et al. 1993). Among active galactic nuclei, this was the only strictly periodic signal reported at any wavelength that passes tests of persistence and phase stability. It has been difficult to understand this behavior.

Madejski et al. (1993) have ended the mystery by showing that, although the period is indeed real, it belongs not to NGC 6814 but to another X-ray source 37 arcmin away in the sky. Follow-up spectroscopy and photometry of several stars in the smaller $\mathrm{X}$-ray error circle revealed the nature of the interloper: a cataclysmic variable, almost certainly of the AM Herculis variety, and provisionally named RX J19402 -1025 (Madejski et, al. 1993; Staubert et al. 1994; $\alpha_{2000}=19^{\mathrm{h}} 40^{\mathrm{m}} 11^{\mathrm{s}} .6, \delta_{2000}^{\prime}=-10^{\circ} 25^{\prime} 25^{\prime \prime} .7$ ).

Although NGC 6814 is now relegated to obscurity, the new X-ray source will surely become one of the most- 

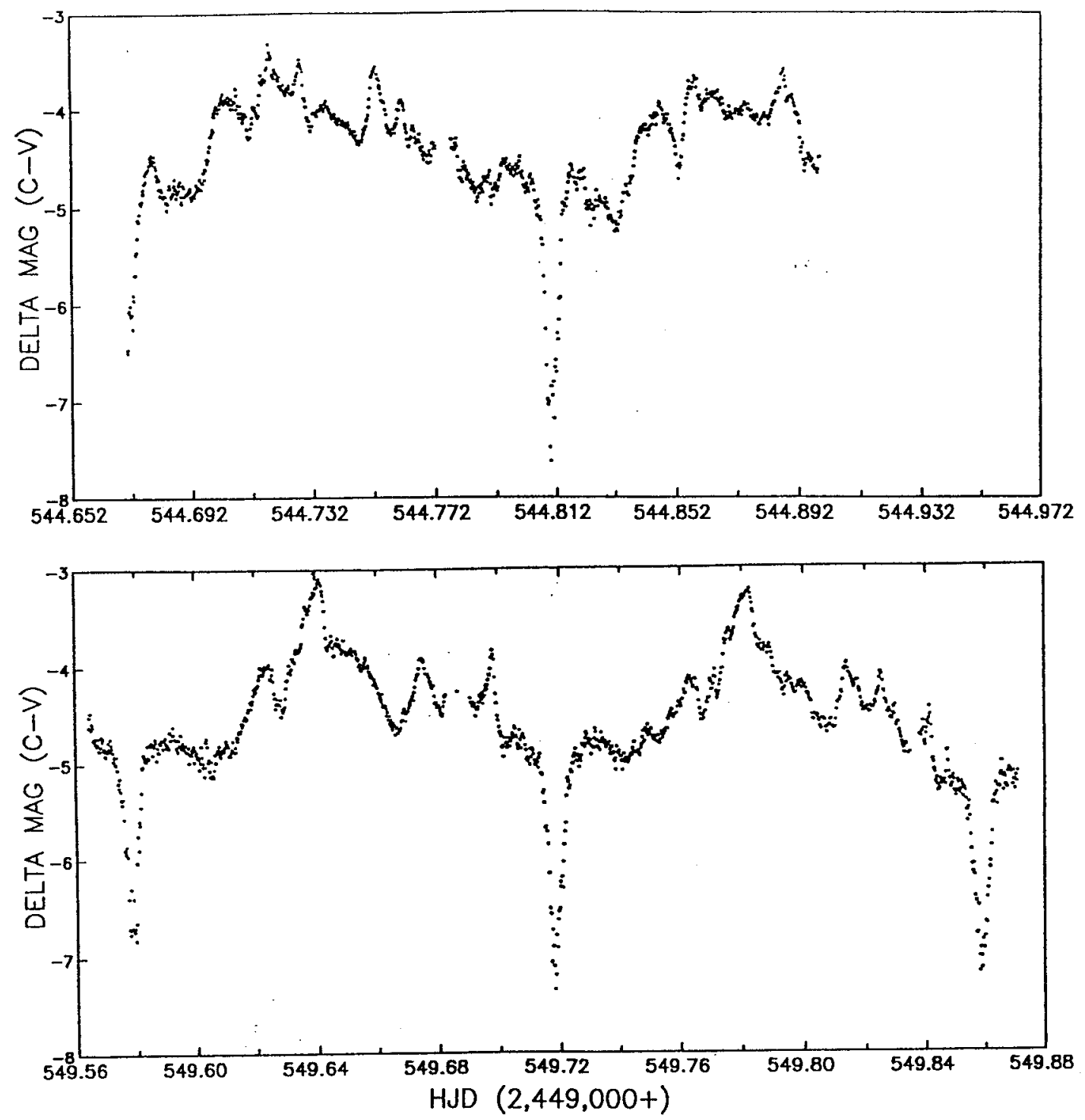

FiG. 1-Photoelectric V-band light curves of RX J19402-1025, obtained in 1994 July. Each point is the average of 2 10-s integrations. A sharp and deep eclipse occurs every $12116 \mathrm{~s}$. A slow and complex wave at a similar period also exists, reaching minimum near the time of the sharp eclipse. Much of what appears to be "flickering" actually repeats with great (but not perfect) fidelity from one orbit to the next.

celebrated and best-studied AM Her stars. One reason is simply that a huge body of X-ray data has already been acquired, motivated by the interest attached to the source when it was presumed to be extragalactic. The other reason is its optical light curve, the principal subject of this paper. Our synoptic photometric observations during 1993-1994 have revealed some quite remarkable features:

(1) sharp and deep eclipses, with a total duration of $\sim 400$ $\mathrm{s}$ and recurring with a strict period of $12116.3 \mathrm{~s}$;

(2) a smooth modulation with the same period; and

(3) a rolling wave which appears to move forward in eclipse phase, showing a fundamental period of $12150 \mathrm{~s}$.

The eclipses and radial velocities demonstrate that the $12116 \mathrm{~s}$ period is the underlying orbital period. The other period (which is closer to previous estimates of the X-ray period) is presumably the white-dwarf's rotation period, since the rotation rate is the rate at which accretion structures near the white dwarf cross our line of sight.
Here we present the evidence for these periods and show that RX J19402-1025 is a "not-quite-synchronous" AM Her star. Precise ephemerides and the presence of sharp eclipses yield a splendid opportunity to learn about the accretion flows in the binary, and the deviation from synchronism even enables the study of how those flows depend on the orientation of the white-dwarf's magnetic field.

\section{LIGHT CURVES}

\subsection{Photoelectric Photometry}

Some of the observations reported here were obtained with the single-channel photometer (ASCAP) mounted on the $1.0 \mathrm{~m}$ telescope at Cerro, Tololo Inter-American Observatory. A focal-plane aperture of 14 arcsec diameter was used, and a Hamamatsu R943-02 photomultiplier tube with a $V$ 
filter. We chose an integration time of $10 \mathrm{~s}$, with an additional dead time of $\sim 1 \mathrm{~s}$ occurring at the end of each integration.

Light curves for two nights of long coverage are shown in Fig. 1. A sharp drop in light occurs every $12116 \mathrm{~s}^{1}$, as some small bright object is eclipsed. ${ }^{3}$ Outside of eclipse, there is a wave of $\sim 1$ mag full amplitude, and some fast variability as well (although much of the latter seems to repeat from one cycle to the next). Close inspection of Fig. 1 suggests that the wave moves slightly in eclipse phase, and this turns out to be true for all of our data: the wave marches towards progressively greater eclipse phase, at the rate of $\sim 0.02$ cycles/day.

\subsection{CCD Photometry}

A larger dataset was obtained with the $\mathrm{CCD}$ photometers on the 0.3 and $0.65 \mathrm{~m}$ "robotic" reflectors of the Center for Basement Astrophysics (Skillman 1993; Skillman and Patterson 1993). The detector was a RCA 504, thinned, backsideilluminated $\mathrm{CCD}$ which was thermoelectrically cooled and actively temperature stabilized at $-35^{\circ} \mathrm{C}$. Exposures were typically 27 - or 87-s long, with an additional $3 \mathrm{~s}$ for detector readout and telescope-pointing corrections. All operations were carried out automatically, since the telescope was unattended. We used a clear WG280 filter, which for a star of this color gives an effective wavelength near $5800 \mathrm{~A}$ (approximately " $V$ "; Skillman and Patterson 1993). Other photometric data were collected with detectors mounted on the CTIO $91 \mathrm{~cm}$ telescope (using a Tek $2048^{2} \mathrm{CCD}$ and V filter) and the McDonald $76 \mathrm{~cm}$ telescope (using a Tek $1024^{2} \mathrm{CCD}$ and I filter).

Table 1 contains the log for all this time-series photometry. After flatfielding and bias subtraction, we removed the sky background and measured differential magnitudes with respect to nearby field stars; this permitted removing the effects of thin cloud.

A dense cluster of CCD light curves is shown in Fig. 2. Again the "flickering" shows a tendency to repeat from one cycle to the next. The out-of-eclipse wave is seen to be shifted by $\sim 0.4$ cycles relative to the appearance in Fig. 1 .

\subsection{Calibration Issues}

With a variety of telescope apertures, detectors, and wavelength sensitivities, we were faced with great heterogeneity in the data; calibration therefore became a serious issue. We measured magnitudes with respect to four comparison stars (which can be seen in Fig. 4 of Staubert et al. 1994):

(a) a "south-following" neighbor star ( 3 " $\mathrm{E}$ and $18^{\prime \prime} \mathrm{S}$ from the variable), which we called comp A;

(b) another "south-following" star (13" E and 3" S away), which we called comp B;

(c) a bright star to the $\mathrm{NE}\left(70^{\prime \prime} \mathrm{E}\right.$ and $110^{\prime \prime} \mathrm{N}$ away), which we called comp $\mathrm{C}$; and

\footnotetext{
${ }^{3}$ To maintain neutrality we generally refer to this as "the eclipse period" or by its numerical value, rather than the interpretation (orbital period) which we shall eventually favor. However, this occasionally compels linguistic awkwardness, and we then lapse into using the interpretative phase. In Sec. 8.1 we will present the evidence favoring that interpretation, and after that we are no longer bashful about it.
}

TABLE 1

Log of Observations

\begin{tabular}{|c|c|c|c|c|c|}
\hline $\begin{array}{l}\text { UT } \\
\text { Date }\end{array}$ & Telescope $^{\mathrm{a}}$ & $\begin{array}{c}(\text { start } \rightarrow \text { end }) \\
\text { HJD }(2440000+)\end{array}$ & $\begin{array}{l}(\mathrm{s}) \\
\Delta t^{\mathrm{b}}\end{array}$ & Pts & Band $^{c}$ \\
\hline $29 \mathrm{Jul} 1993$ & 1 & $9197.64680-0.68533$ & 90 & 37 & $\mathrm{C}$ \\
\hline 31 Jul 1993 & 1 & $9199.58010-0.71136$ & 90 & 98 & $\mathrm{C}$ \\
\hline 3 Aug 1993 & 1 & $9202.57270-0.63313$ & 90 & 47 & C \\
\hline 9 Aug 1993 & 1 & $9208.54552-0.59761$ & 90 & 29 & C \\
\hline 10 Aug 1993 & 1 & $9209.55583-0.74751$ & 90 & 161 & $\mathrm{C}$ \\
\hline 7 Sep 1993 & 1 & $9237.56144-0.66664$ & 90 & 80 & $\mathrm{C}$ \\
\hline 7 Sep 1993 & 2 & $9237.59263-0.72551$ & 40 & 187 & I \\
\hline 8 Sep 1993 & 2 & $9238.60344-0.77240$ & 32.5 & 518 & I \\
\hline 11 Sep 1993 & 1 & $9241.51229-0.65605$ & 90 & 69 & $\mathrm{C}$ \\
\hline $12 \operatorname{Sep} 1993$ & 1 & $9242.51221-0.65493$ & 90 & 132 & $\mathrm{C}$ \\
\hline $14 \operatorname{Sep} 1993$ & 1 & $9244.50681-0.65057$ & 90 & 136 & $\mathrm{C}$ \\
\hline 29 Sep 1993 & 1 & $9259.50846-0.60327$ & 90 & 63 & $\mathrm{C}$ \\
\hline 4 Oct 1993 & 1 & $9264.49035-0.59245$ & 90 & 99 & $\mathrm{C}$ \\
\hline 5 Oct 1993 & 1 & $9265.52569-0.59029$ & 90 & 63 & $\mathrm{C}$ \\
\hline 6 Oct 1993 & I & $9266.47874-0.58812$ & 90 & 94 & $\mathrm{C}$ \\
\hline 11 Oct 1993 & 1 & $9271.47731-0.57106$ & 90 & 91 & C \\
\hline 13 Oct 1993 & 1 & $9273.47715-0.56465$ & 90 & 84 & $\mathrm{C}$ \\
\hline 11 Nov 1993 & 1 & $9302.45459-0.49002$ & 90 & 28 & $\mathrm{C}$ \\
\hline 12 Nov 1993 & 1 & $9303.45034-0.47014$ & 90 & 14 & $\mathrm{C}$ \\
\hline 23 Apr 1994 & 3 & $9465.82907-0.89921$ & 30 & 203 & $\mathrm{C}$ \\
\hline 11 May 1994 & 3 & $9483.81545-0.88317$ & 30 & 195 & $\mathrm{C}$ \\
\hline 28 May 1994 & 3 & $9500.77449 .0-86929$ & 30 & 239 & C \\
\hline 3 Jun 1994 & 3 & $9506.74987-0.87072$ & $\cdot 30$ & 349 & C \\
\hline 10 Jun 1994 & 3 & $9513.73955-0.79095$ & 30 & 149 & C \\
\hline $10 \mathrm{Jul} 1994$ & 4 & $9543.84839-0.91284$ & 11.028 & 505 & V \\
\hline 11 Jul 1994 & 4 & $9544.67025-0.89838$ & 11.061 & 1748 & V \\
\hline I2 Jul 1994 & 4 & $9545.63289-0.89714$ & 11.121 & 993 & V \\
\hline I6 Jul 1994 & 4 & $9549.56436-0.87460$ & 11.081 & 2333 & $\mathrm{v}$ \\
\hline 24 Jul 1994 & 6 & $9557.75691-0.89576$ & 107,47 & 113 & v \\
\hline 27 Jul 1994 & 6 & $9560.79247-0.83782$ & 32 & 111 & $\mathrm{v}$ \\
\hline 29 Jul 1994 & 6 & $9562.67598-0.71197$ & 77 & 35 & $\mathrm{v}$ \\
\hline 7 Aug 1994 & 3 & $9571.57125-0.71570$ & 30 & 417 & $\mathrm{C}$ \\
\hline 8 Aug 1994 & 3 & $9572.56220-0.72851$ & 30 & 376 & $\mathrm{C}$ \\
\hline 13 Aug 1994 & 2 & $9577.66400-0.90926$ & 34 & 402 & v \\
\hline I4 Aug 1994 & 2 & $9578.62255-0.89721$ & 34 & 664 & v \\
\hline 15 Aug 1994 & 2 & $9579.63740-0.88707$ & 34 & 550 & $\mathrm{~V}$ \\
\hline 16 Aug 1994 & 2 & $9580.61866-0.89588$ & 34 & 573 & V \\
\hline 17 Aug 1994 & 2 & $9581.60973-0.88514$ & 34 & 659 & $\mathbf{V}$ \\
\hline 18 Aug 1994 & 2 & $9582.64187-0.89108$ & 34 & 538 & V \\
\hline 19 Aug 1994 & 3 & $9583.54133-0.66864$ & 25.3 & 241 & $\mathrm{C}$ \\
\hline 3 Sep 1994 & 3 & $9598.55533-0.65985$ & 30 & 300 & C \\
\hline $9 \operatorname{Sep} 1994$ & 3 & $9604.51595-0.65102$ & 30 & 382 & $\mathrm{C}$ \\
\hline
\end{tabular}

Notes to TABLE 1

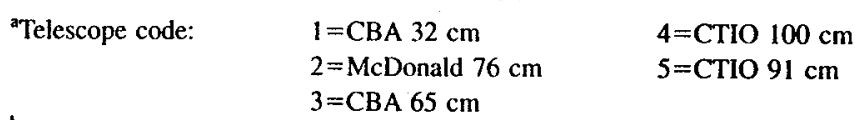

${ }^{\mathrm{b}} \Delta t=$ integration time plus dead time; other gaps in the time series exist due to background measurement, editing of bad points, etc.

'Bandpasses: $\quad \mathrm{V}=$ Johnson $V \quad \mathrm{I}=\mathrm{J}$ ohnson $I$

$\mathrm{C}=$ Unfiltered on $\mathrm{CBA}$ instrumental scale (approximately $V$ )

(d) another star to the NE (40" E and 50" N), which we called comp D.

Photometry on several clear nights revealed magnitudes given in Table 2 . We primarily used comp $\mathrm{C}$ for the CBA photometry, and adjusted other data streams to give consistent values for comp,$C$. Variety in passband was a problem, though, and we estimate that the different datasets may contain systematic errors of $\sim 0.1$ mag in zero point.

On good nights with good calibration, RX J19402-1025 

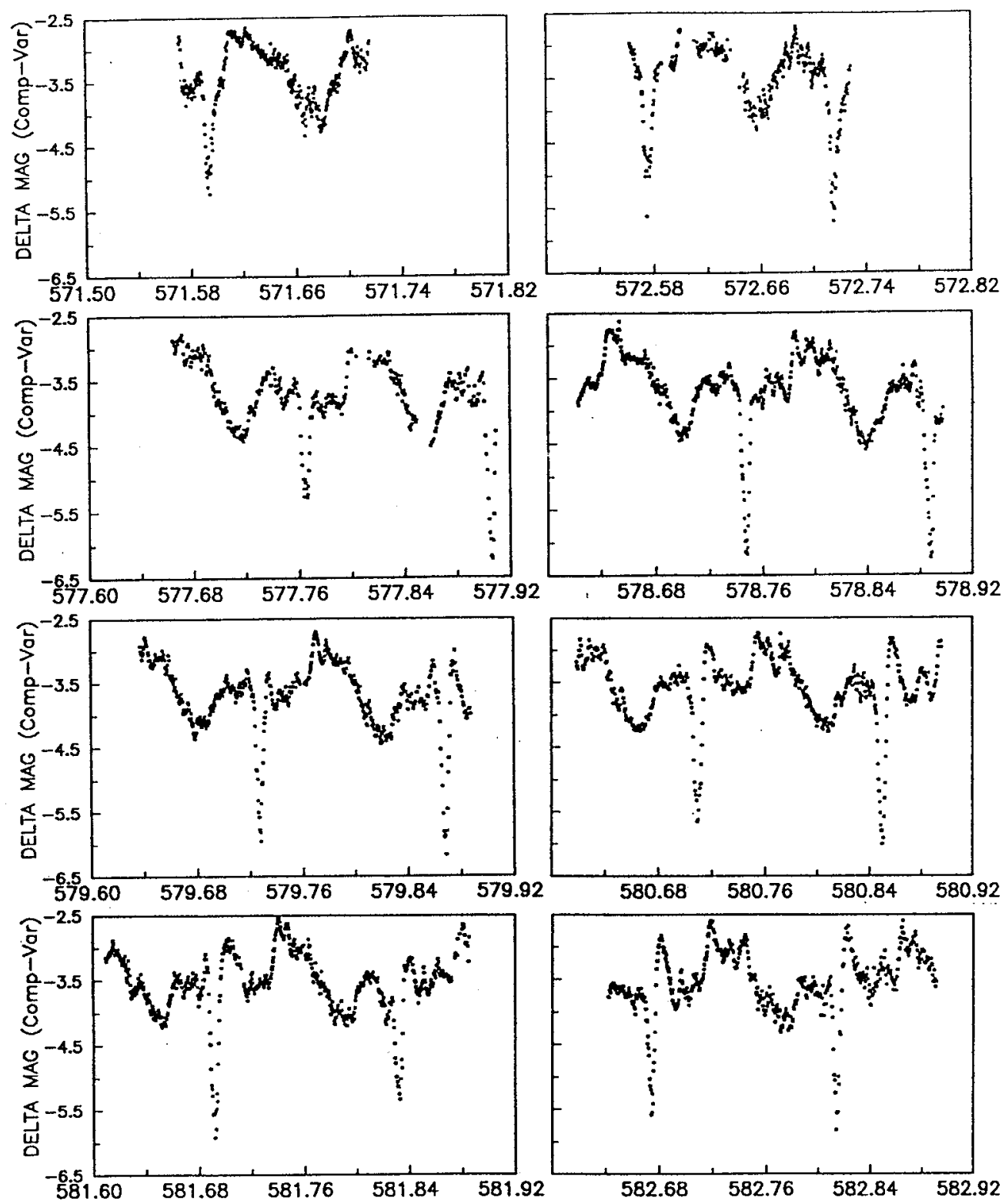

HJD $2,449,000+$

FIG. 2-CCD V-band light curves of RX J19402-1025, obtained in 1994 August. Each point is a 20-s integration, with $14 \mathrm{~s}$ of dead time, giving an effective time resolution of $34 \mathrm{~s}$. Comparing to Fig. 1 , note that the slow wave has shifted its phase by $\sim 0.4$ cycles relative to the sharp eclipse. Again there is a tendency for the flickering to repeat fairly well from one cycle to the next.

TABLE 2

Magnitudes of RX1940.2-1025 and Comparison Stars

\begin{tabular}{lccccc}
\hline \hline \multicolumn{1}{c}{ Star } & $B$ & $V$ & $R$ & $I$ & Clear $^{2}$ \\
\hline Comp A & 15.97 & 15.06 & 14.50 & 13.91 & 3.46 \\
Comp B & 16.69 & 15.64 & 15.10 & 14.53 & 4.04 \\
Comp C & $\cdots$ & $\cdots$ & $\cdots$ & $\cdots$ & 0.00 \\
Comp D & $\ldots$ & $\ldots$ & $\cdots$ & $\cdots$ & 3.31 \\
RX J19402-1025 & & & & & \\
(out of eclipse) & $\cdots$ & $14.9-16.0$ & $\cdots$ & $\cdots$ & $3.4-5.0$ \\
RX J19402-1025 & & & & & \\
(mid-eclipse) & $\cdots$ & $\sim 18$ & $\cdots$ & $\cdots$ & $\sim 7$ \\
\hline
\end{tabular}

Notes to TABLE 2

a"clear" refers to differential magnitudes on the unfiltered CBA system, roughly equivalent to $\Delta V$ (Skillman and Patterson 1993). The zero point is here determined by setting the clear magnitude of comp B equal to $V+0.5(V-R)$. typically varied from $V=14.8$ to 16.0 outside of eclipse, and dropped to 18 in mid-eclipse. The star rose to $V=14.2$ in its brightest flares.

\section{PERIOD ANALYSIS}

\subsection{The Eclipse Period}

The eclipse period is easily studied, as the feature in the light curve is very sharp. We define mid-eclipse as the mean of the times of mid-ingress and mid-egress (measured from units of intensity, not magnitudes), and present in Table 3 the measured mid-eclipse times. $A^{\prime}$ linear fit to these timings, along with those reported by Watson et al. (1995) and Koenig (1994), yields an ephemeris 
TABLE 3

Times of Mid-Eclipse

\begin{tabular}{rccc}
\hline $\begin{array}{r}\text { Cycle } \\
\text { Number }\end{array}$ & $\begin{array}{c}\text { (HJD 2440000+) } \\
\text { Mid-eclipse }\end{array}$ & $\begin{array}{c}\text { Cycle } \\
\text { Number }\end{array}$ & $\begin{array}{c}\text { (HJD 2440000+) } \\
\text { Mid-eclipse }\end{array}$ \\
\hline & & & \\
0 & 9199.6957 & 2496 & 9549.7188 \\
71 & 9209.6487 & 2652 & 9571.5930 \\
278 & 9238.6772 & 2659 & 9572.5753 \\
299 & 9241.6210 & 2660 & 9572.7158 \\
306 & 9242.6031 & 2696 & 9577.7650 \\
320 & 9244.5677 & 2703 & 9578.7467 \\
385 & 9253.6836 & 2704 & 9578.8871 \\
406 & 9256.6300 & 2710 & 9579.7277 \\
427 & 9259.5741 & 2711 & 9579.8674 \\
512 & 9271.4959 & 2717 & 9580.7096 \\
1898 & 9465.8591 & 2718 & 9580.8501 \\
2147 & 9500.7776 & 2724 & 9581.6919 \\
2190 & 9506.8068 & 2725 & 9581.8321 \\
2461 & 9544.8107 & 2731 & 9582.6747 \\
2467 & 9545.6513 & 2732 & 9582.8149 \\
2495 & 9549.5782 & 2887 & 9604.5507 \\
\hline \hline
\end{tabular}

Mid-eclipse $=$ HJD $2449199.6931+0.1402345 E$.

Figure 3 shows the scatter of eclipse timings with respect to this ephemeris, with open circles indicating measurements of lower weight (usually because the light curve was of inferior time resolution). The rms scatter is $\sim 60 \mathrm{~s}$, marginally greater than the estimated measurement error of $40 \mathrm{~s}$.

A selection of light curves near eclipse is shown in Fig. 4. From these and others we measured eclipse ingress and egress times, and the eclipse width reckoned from the "halfpower points" (mid-ingress and mid-egress). The eclipse width averaged $632 \pm 35 \mathrm{~s}$, and a linear fit to ingress and egress yielded 341 and $311 \mathrm{~s}$, respectively. ${ }^{4}$

\subsection{The Slow Modulation at the Eclipse Period}

In order to study slow variations in the light curve, we merged all the 1993 data, after degrading the time resolution of the 8 September light curve to approximate that of the CBA light curves. We also measured and digitized the light curves in unfiltered (approximately blue) light published by Watson et al. (1995). We normalized these measured light curves to give the same light level (in delta magnitudes) as the mean CBA light curve. The same was done for the I-band observations of 1993 September 7 and 8. This procedure is correct only if the waveform is approximately constant from blue to infrared wavelengths, and if there are no large changes in the mean light level. Direct inspection of the light curves supported the first condition, and the CBA coverage supported the second, since that coverage spanned all of the other data and showed no large ( $>0.5 \mathrm{mag}$ ) changes in mean brightness.

\footnotetext{
${ }^{4} \mathrm{But}$ we caution that the ingress/egress estimates are quite difficult to make and even more difficult to interpret, because the "linear fit" is not really defensible in the presence of strong erratic variability (since the light-level change associated with first and second contact is, in principle, zero!). That is why we are reluctant to estimate errors; the intrinsic scatter is $\sim 80 \mathrm{~s}$.
}

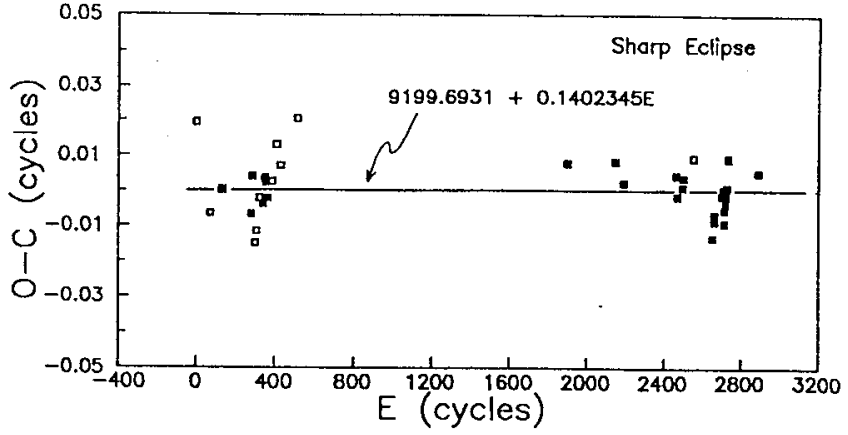

FIG. 3-O-C diagram of the timings of mid-eclipse with respect to Eq. (1). Filled squares are estimated to have a measurement error of $30 \mathrm{~s}$; open squares are estimated to have an error $\sim 60 \mathrm{~s}$. There appears to be some scatter beyond measurement etror, but generally the sharp eclipse is a very good clock indeed.

The resultant light curve spanned 106 days. While fairly sparse (77 hr spread over $31 \mathrm{~d}$ ), the light curve is welldistributed: a spread in observatory longitude subdued oneday aliases, the dedication of the CBA telescope prevented aliasing with the lunar phase, and by good fortune there is one very dense segment of data (10 nights covered during September 7-19).

Finally, we removed the sharp eclipses from the data, either outright or by inserting dummy points interpolated across the eclipse (when the light levels before and after eclipse were well-defined). This was advisable because sharp features are anathema to Fourier transforms, especially with deep eclipses rendered on a magnitude scale.

We then calculated the power spectrum from the discrete Fourier transform of the light curve, with the result shown in the lower panel of Fig. 5. The dominant peak occurs at $12117 \pm 3 \mathrm{~s}$. The upper panel shows the power spectrum of an artificial light curve containing a pure sinusoid at this period, sampled exactly like the actual data. This shows the obvious one-day aliases, but not the next-highest peak in the lower panel-which occurs at $6075 \pm 2 \mathrm{~s}, 6 \sigma$ removed from the first harmonic of the primary signal. The only other features of momentary interest occur at 11.179 and $21.333 \mathrm{c} / \mathrm{d}$. The former appears in the upper panel, and disappears after the primary signal is removed (as we shall find below); this indicates an origin in aliasing. The latter is not an alias, but we shall soon see that it is the second harmonic of a fundamental frequency in the data.

Since the primary signal occurs at a period very close to and consistent with the precisely known eclipse period, we used the latter to perform a synchronous summation and find the mean wave form of the primary signal. The result is seen in Fig. 6. Basically the light curve is flat for about half an orbit, slowly dips to a minimum centered on eclipse phase $0.98 \pm 0.02$, then recovers slightly faster. Neither the asymmetry nor the secondary dip at phase 0.5 should be regarded as established, because the other signal at $6075 \mathrm{~s}$ is present and does not equally populate all phase bins.

On the assumption that this slow modulation at $12116 \mathrm{~s}$ is persistently present in the data (unproven, but the data are consistent with it), we then subtracted this signal from the season-long light curve. More specifically, we subtracted the 

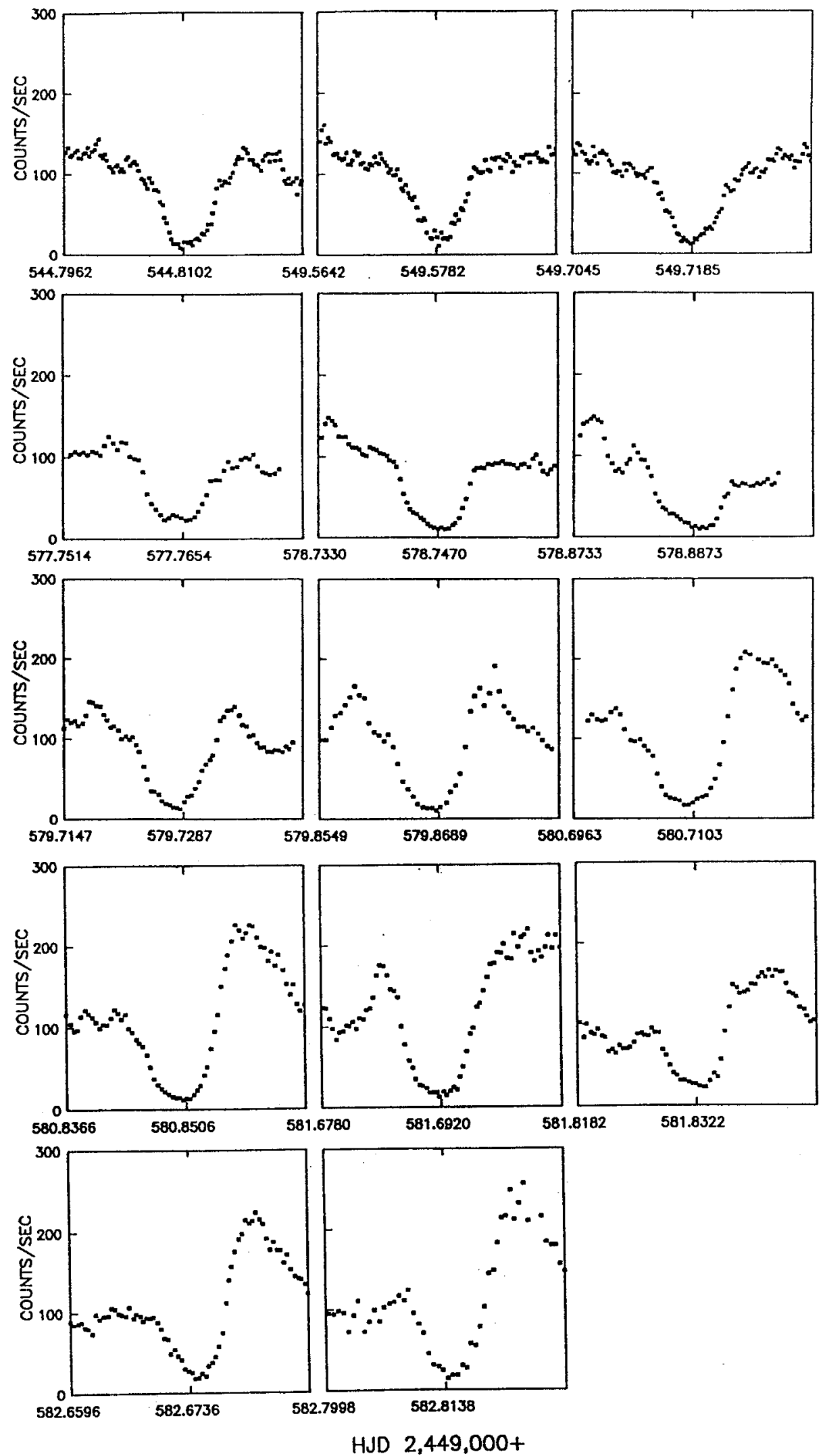


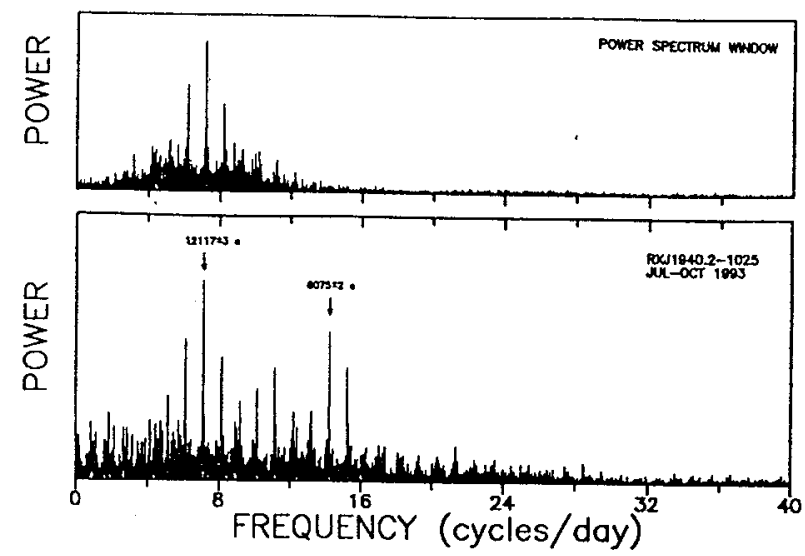

Fig. 5-Lower frame, power spectrum of the 1993 light curve, after removing the sharp eclipses. A strong signal at $12117 \pm 3 \mathrm{~s}$ is obvious. Upper frame, power spectrum of an artificial light curve containing a sinusoidal signal at this period, sampled exactly as the actual light curve. All of the secondary peaks seen in the lower frame appear and are therefore aliases of the primary signal, except for the $6075 \pm 2$-s signal marked with an arrow. The latter is therefore a real and independent periodicity.

best-fit sinusoid at the independently known eclipse period. This produces a large change in the nightly light curves, and should be remembered when comparing the results described below to the doubtless rich crop of future results on this star.

\subsection{The Second Period}

Our earliest observations of this star suggested a period $(\sim 12150 \mathrm{~s}$; Patterson et al. 1993) considerably longer than the eclipse period. This still appears to be true, but in a rather complex manner. We recalculated the power spectrum after subtracting the primary signal as described above, with the result seen in Fig. 7. A strong signal appears at $6075 \mathrm{~s}$, as evident in the previous power spectrum and in the raw light curve. We have designated this signal " $1 \mathrm{H}$ " to indicate that it is the first harmonic of an unseen fundamental signal at $12150 \mathrm{~s}$. [Although no power appears at $12150 \mathrm{~s}$, that is evidently the fundamental signal, because Fig. 7 shows a set of frequencies which are strict harmonics of $12150 \mathrm{~s}$, but not

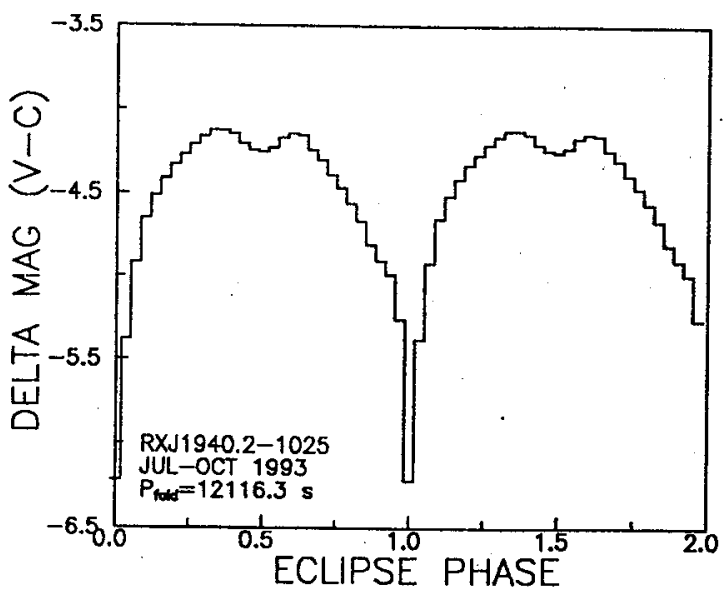

FIG. 6-The mean 1993 light curve, synchronously summed on the eclipse period. The slow variation at this period reaches minimum light at or slightly before the sharp eclipse. This light curve is uncorrected for effects of the 6075-s signal.

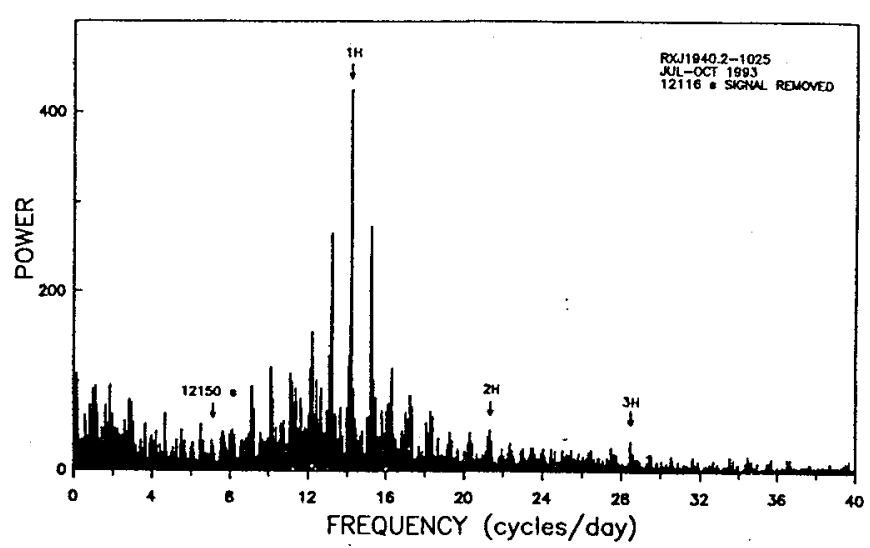

FIG. 7-Power spectrum of the 1993 light curve, after subtracting the $12,116-s$ signal. The only obvious peak occurs at $14.222(2) \mathrm{c} / \mathrm{d}(P=6075 \mathrm{~s})$, but other significant peaks occur at $21.333(3)$ and $28.443(3) \mathrm{c} / \mathrm{d}$. We interpret these as harmonics of an unseen fundamental frequency of $7.111 \mathrm{c} / \mathrm{d}$ $(P=12150 \mathrm{~s})$. Arrows point to these harmonics.

$6075 \mathrm{~s}$. For example, there is a significant peak at 21.333 (3) $\mathrm{c} / \mathrm{d}$, an exact harmonic of an unseen 12150-s signal, but not of the obvious large signals at 12116 and $6075 \mathrm{~s}$.]

We then synchronously summed the 1993 light curve to produce the mean wave form of the $12150 \mathrm{~s}$ variation. This is shown in the lower frame of Fig. 8. Moderate evidence for a double-humped waveform appears, with alternating broad and narrow maxima. Arbitrarily defining the (very slightly)
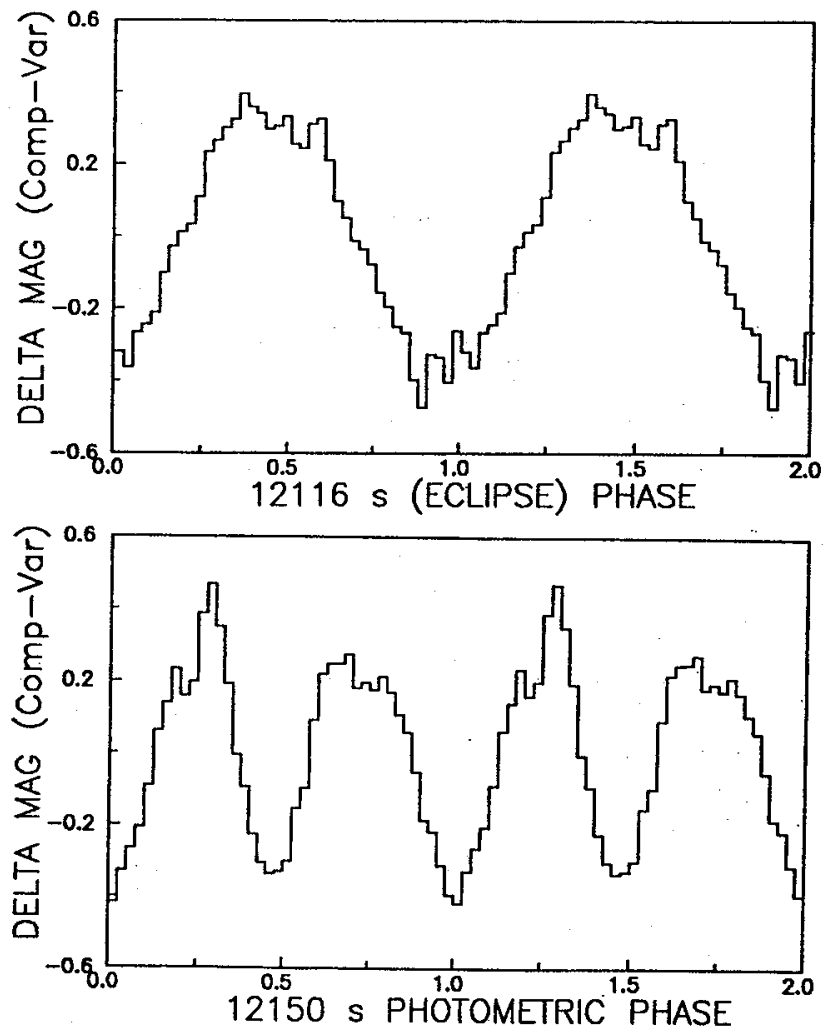

Fig. 8-Lower frame, mean 1993 light curve folded on the 12150-s period, showing moderate evidence' for a pattern of alternating broad and narrow maxima. Upper frame, mean 1993 light curve folded on the 12116-s period, bridging over the sharp eclipses. In both cases the light curve was first "prewhitened" to remove the contaminating effect of the other signal. 

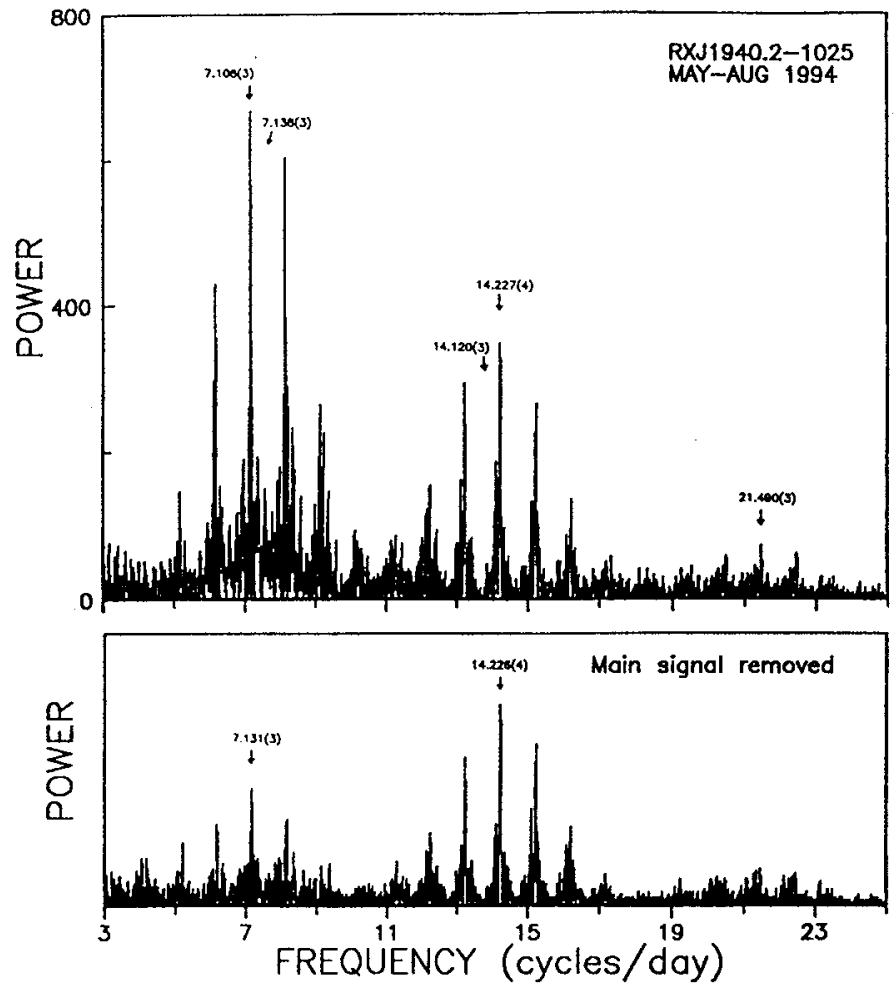

FIG. 9-Upper frame, power spectrum of the 1994 light curve. Significant peaks are labeled with their frequencies in c/day; other peaks arise from the sampling pattern ("power spectrum window"). Lower frame, power spectrum after subtracting the dominant 12150-s signal. Note that the "7.138 $\mathrm{c} / \mathrm{d}$ " signal moves to $7.131 \mathrm{c} / \mathrm{d}$, the signal familiar and dominant in the 1993 data (the location in the upper frame was altered by aliasing of the main signal).

deeper minimum as phase zero, the variations obey the ephemeris

Prim. $\min .=$ HJD $2449197.7430+0.140625 E$.

The "secondary minimum" occurs at phase $0.466(9)$, the lower and broad maximum at $0.708(20)$, and the higher and narrow maximum at $0.238(16)$.

Of course, the 12150/6075-s signal corrupts the timings of the slow variation at the eclipse period (though not the eclipse itself, which is too sharp to be seriously affected). In order to study the 12116-s signal, we subtracted the 12150-s wave form from the original light curve sans eclipses, and synchronously summed on the 12116-s period. The result is shown in the upper frame of Fig. 8, indicating a very nearly sinusoidal wave form with minimum light occurring at eclipse phase $0.949(13)$. This suggests that the precise details of the waveform shown in Fig. 6 are unreliable, probably arising from contamination by the $12150 / 6075$-s signal.

\section{1994 PHOTOMETRY}

We analyzed the 1994 photometry separately. The power spectrum of the seasons's light curve is shown in the upper panel of Fig. 9, and detailed examination shows striking differences. The strongest peaks occur at 7.108(3) and 7.138(3) c/d. These appear to be the same signals found in 1993 (although the power at $12150 \mathrm{~s}$ exactly is now dominant rather
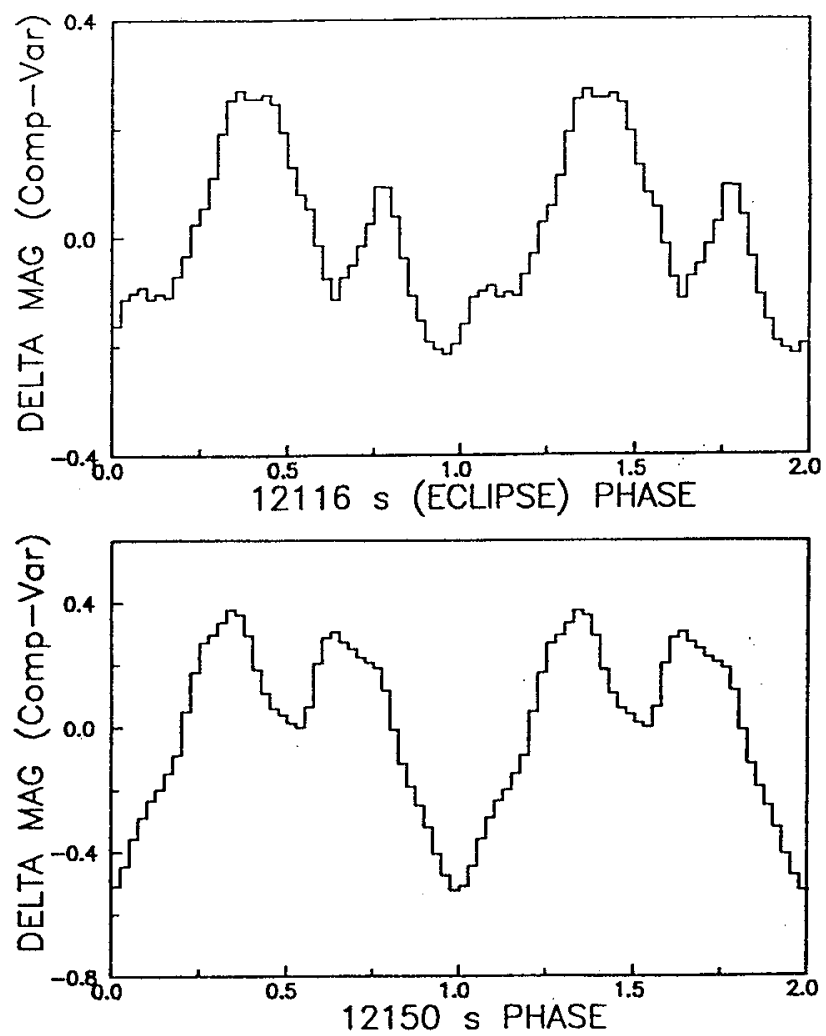

Fig. 10-Upper frame, mean 1994 light curve folded on the 12116-s period [Eq. (1)], bridging over the sharp eclipses. Lower frame, mean 1994 light curve folded on the 12150-s period [Eq. (3)]. In both cases the light curve was first prewhitened to try to remove the contaminating effect of the other signal.

than undetectable!). Another peak occurs at 14.227(4) c/d, probably the first harmonic of the "12150-s" signal. The arrows in Fig. 9 point to other significant and somewhat mysterious peaks at $14.120(3)$ and $21.490(3) \mathrm{c} / \mathrm{d}$.

We also "CLEANed" the power spectrum in the following sense. We subtracted the strongest signal from the light curve and recalculated the power spectrum, with the result seen in the lower panel of Fig. 9. ${ }^{5}$ The highest peak now occurs at $12108 \pm 7 \mathrm{~s}$, indicating that the 12116-s signal is indeed still present in the data.

We proceeded to analyze the three strongest signals, measuring phases and amplitudes. The $6075 \mathrm{~s}$ signal has a full amplitude of $0.37 \mathrm{mag}$ and reaches minimum light at 9506.7692. ${ }^{6}$ The 12150-s signal needs a fuller discussion. The waveform, shown in the lower frame of Fig. 10, indicates that a deep minimum is the primary distinguishing fea-

\footnotetext{
5This is related to the strategy of the popular "CLEAN" algorithm for periodfinding (Roberts et al. 1987). It has very severe drawbacks when applied too vigorously, because it can disable the human being's skill, finely honed over eons of time, at estimating the noise level. But for the removal of one strong signal in a fairly well-sampled time series, the noise is not too badly suppressed. We use it for one purpose only: to inspect the frequency neighborhood of a strong signal. Unlike the popular algorithm, we simply remove the strong signal (rather than adding it back without its family of aliases).

${ }^{6}$ To paraphrase Lord Acton, all numbers clutter text, and absolute numbers clutter absolutely. We try to preserve some readability by dropping the assumed "HJD 2440000 +" in most cases.
} 
TABLE 4

Log of Spectroscopic Observations

\begin{tabular}{rccc}
\hline \hline date (UT) & UT Range & phase range & eclipse $^{\mathrm{a}}$ \\
\hline 1994 Sep 23 & $3: 04-6: 03$ & $0.622-1.510$ & 9253.6835 \\
Sep 24 & $2: 40-2: 50$ & $0.635-0.687$ & (b) \\
Sep 25 & $3: 14-3: 57$ & $0.932-1.146$ & 9255.6464 \\
Sep 26 & $2: 39-5: 26$ & $0.890-1.731$ & 9256.6279 \\
Sep 27 & $2: 22-3: 07$ & $0.935-1.161$ & 9257.7610 \\
Sep 29 & $4: 20-5: 53$ & $0.778-1.240$ & (c) \\
\hline
\end{tabular}

Notes to TABLE 4

${ }^{a}$ Heliocentric JD of mid-eclipse, minus 2440000 .

${ }^{b}$ Cut short because source was too close to moon.

'Apparent guiding error; no eclipse timing.

ture; it occurs at 9506.8380 , which corresponds to phase 0.01 on the 1993 ephemeris for this signal. The improved ephemeris spanning both years is

Primary minimum $=9197.7430+0.1406256 E$.

The other features in the double-humped 12,150-s wave form may not be stable. The secondary minimum occurred at phase $0.466(11)$ and $0.515(13)$ in 1993 and 1994, respectively. "Primary" maximum (following the deep minimum) occurred at phase $0.247(13)$ in 1993 and $0.328(15)$ in 1994, while secondary maximum occurred at phase $0.717(17)$ in 1993 and $0.698(24)$ in 1994.

The 12116-s signal has a full amplitude of $0.38 \mathrm{mag}$ and reaches minimum light at 9506.7996 , which corresponds to eclipse phase $0.949(23)$. The waveform, shown in the upper frame of Fig. 10, is significantly different from that of 1993 (Fig. 8). In 1994 the star displayed an amplitude only half as large, and a pronounced $\sim 0.2 \mathrm{mag}$ dip near phase 0.6 .

\section{THE LONGER PERIOD AND THE SUPERCYCLE}

We also calculated the power spectrum for the entire 1993-4 light curve. This yielded peaks at 12150.3 and $12147.8( \pm 0.2) \mathrm{s}$, and between these we could not establish a preference. A half-cycle of phase difference between these candidate clocks accumulates per year; normally this would be easy to distinguish, but not here because of the doublehumped wave form in 1993.

The two basic clocks in the star beat together with a period $\left[=\left(\nu_{1}-\nu_{2}\right)^{-1}\right]$ of $50.4 \pm 0.2$ days if the longer period is $12150.3 \mathrm{~s}$, or 54.1 days if the longer period is $12147.8 \mathrm{~s}$. Thus the photometric waveform should repeat on this "supercycle." The evident variability on very short (5-30 min) and very long $(\sim y r)$ time scales ensures that this will not exactly be true, but there should be a very marked pattern repeating on this period.

\section{SPECTROSCOPIC OBSERVATIONS AND ANALYSIS}

\subsection{New Observations}

We carried out spectroscopic observations with the Hiltner $2.4 \mathrm{~m}$ reflector at Michigan-Dartmouth-MIT (MDM) Observatory on Kitt Peak. The Mark III grism spectrograph,

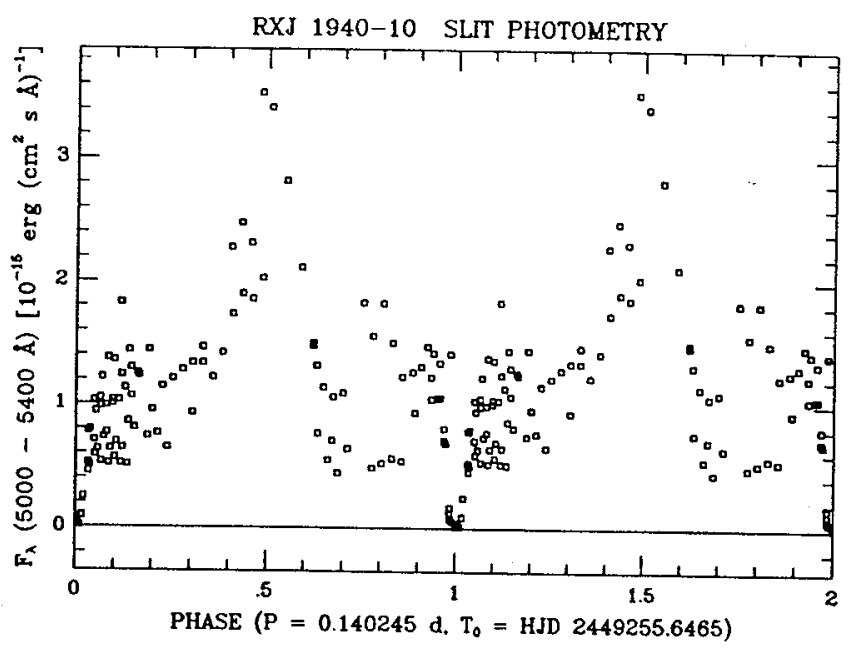

FIG. 11-Continuum fluxes (5000-5400 A) from the spectroscopic measurements, phased on the local ephemeris. Data are shown twice for clarity. A deep eclipse occurs on schedule, and a sharp maximum near phase 0.5 .

a $600 \mathrm{l} / \mathrm{mm}$ grism, and a Tektronix $1024^{2} \mathrm{CCD}$ gave 2.2 A/pixel from 4420 to $6670 \mathrm{~A}$. The $1.2^{\prime \prime}$ slit yielded a (slightly undersampled) spectral resolution of $4 \mathrm{~A}$ FWHM. We rotated the instrument frequently to keep the slit near the parallactic angle for all observations. The seeing was very good (FWHM $<1^{\prime \prime}$ ) most of the time, and the weather was generally clear but not always photometric. We used exposure times of 5 or $8 \mathrm{~min}$, but shortened them to $3 \mathrm{~min}$ in eclipse. All exposures were flanked by $\mathrm{HgNeXe}$ exposures, and the dead time between exposures was $16 \mathrm{~s}$. The spectroscopic observing log is given in Table 4.

We used standard IRAF routines to reduce the data through bias subtraction, flatfielding, and reduction to onedimensional spectra. The wavelength solutions had typical residuals $<0.1 \mathrm{~A}$. As an end-to-end check, we measured the apparent velocity of the $\lambda 5577$ night-sky line in all the sky spectra; its mean was $+3.7 \mathrm{~km} \mathrm{~s}^{-1}$, with a standard deviation of $2.1 \mathrm{~km} \mathrm{~s}^{-1}$. We divided the spectra by the spectrum of a hot star to remove residual instrumental effects, and used standard-star observations to convert to absolute flux units.

The observations define the eclipse center with $\sim 40 \mathrm{~s}$ accuracy. Timings of mid-eclipse are given in Table 4; from these we derive a local eclipse epoch HJD 2449255.64646. This departs from the precise ephemeris adopted above by only $24 \mathrm{~s}$, hence is satisfactory for all purposes. The mean light curve for the week of observations, based on the $5000-$ 5400 A continuum region, is shown in Fig. 11. The eclipse is plainly evident, and a large maximum near orbital phase 0.5 .

In order to establish the radial-velocity period independently, we measured rough radial velocities of the $\mathrm{H} \alpha$ line using the Gaussian-convolution method (Schneider and Young 1980) optimized for 32 A FWHM; this effectively gave a "whole-line" center. Of our 108 spectra, 94 gave useful results. A sinusoidal fit to the velocities gave a period of 12122(9) s, with an epoch (blue-to-red crossing) of 9255.6254. However, the radial-velocity curve from the whole-line fit (not shown) departs greatly from a sinusoid, so we do not attribute any physical significance to this epoch. 


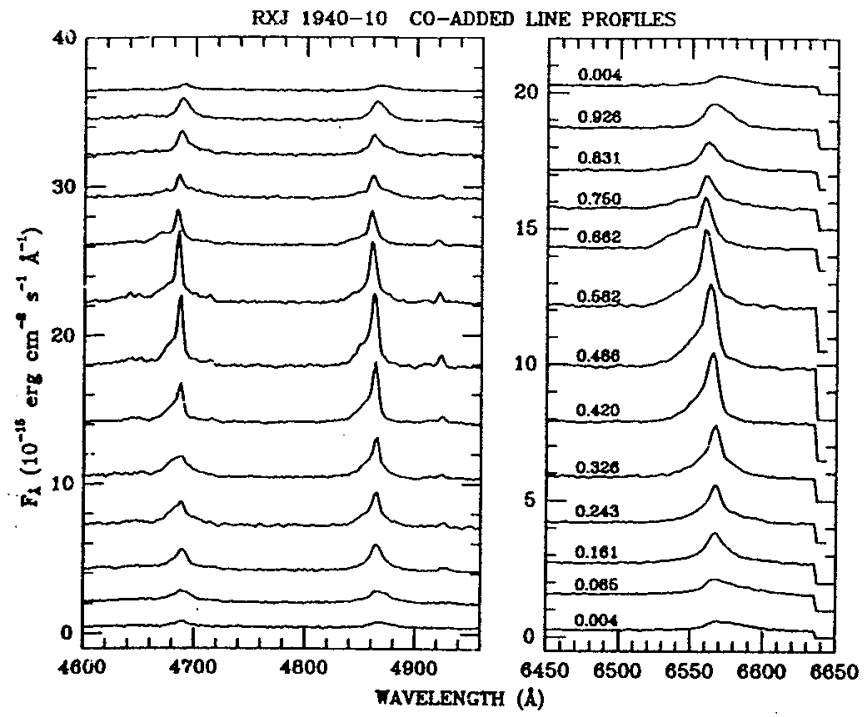

Fig. 12-Line profiles after coadding in phase. The right-hand panel shows $\mathrm{H} \alpha$, and the label on each trace gives the phase. Time increases from the bottom, and the top trace is a repeat of the bottom trace. Spectra are offset vertically to avoid overlap. The left-hand panel is similar but with horizontal and vertical scales adjusted to the region of $\mathrm{He}$ II 4686 and $\mathrm{H} \beta$.

To study spertral changes around the orbit at improved signal to noise, we coadded the spectra into phase bins using two different binning schemes. In one we used 12 equal phase bins, with one bin centered on the eclipse; in the other we used 15 3-min bins centered on eclipse in order to match the binning to our best time resolution. The phase assigned to each bin was the average of the phases of the included spectra. Figure 12 shows the full-orbit phase-binned spectra in the regions of $\mathrm{He}$ II $\lambda 4686, \mathrm{H} \beta$, and $\mathrm{H} \alpha$; Figure 13 shows the high time-resolution spectra near eclipse.

Figures 12 and 13 show that the complex line profiles make radial-velocity measurement problematical. This is a well-known problem in AM Her stars. Physical models of the line profiles have a dauntingly large number of param-

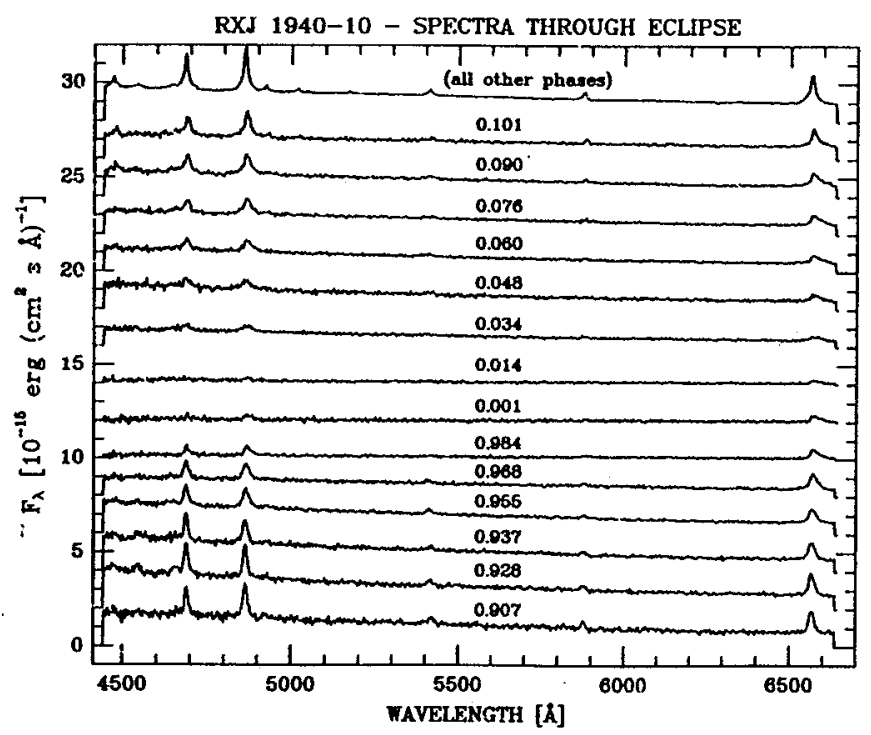

Fig. 13-Coadditions of spectra near eclipse; the bins are nominally $180-\mathrm{s}$ apart, with some overlap due to the lengths of the exposures. Note that the emission-line eclipse is centered at a time slightly later (by $\sim 0.014$ in phase) than the continuum eclipse. The top trace shows the sum of all the out-ofeclipse spectra.
TABLE 5

Components of the $\mathrm{H} \alpha$.Line

\begin{tabular}{lrrcrrc}
\hline \hline \multirow{2}{*}{ Phase } & \multicolumn{3}{c}{ Sharp Component } & \multicolumn{3}{c}{ Broad Component } \\
& \multicolumn{1}{c}{$V$} & Flux & FWHM & \multicolumn{1}{c}{$V$} & Flux & FWHM \\
\hline 0.004 & 270 & 1.9 & 635 & 633 & 9.8 & 1835 \\
0.085 & 176 & 4.1 & 672 & 616 & 15.9 & 2145 \\
0.161 & 170 & 8.9 & 545 & 237 & 18.2 & 2031 \\
0.243 & 161 & 7.0 & 361 & 133 & 22.8 & 1715 \\
0.326 & 159 & 9.2 & 368 & 55 & 27.7 & 1535 \\
0.420 & 75 & 14.9 & 372 & -227 & 28.7 & 1203 \\
0.468 & 1 & 18.5 & 383 & -316 & 37.4 & 1359 \\
0.582 & -134 & 17.3 & 381 & -391 & 36.1 & 1596 \\
0.662 & -159 & 9.68 & 346 & -561 & 32.0 & 1742 \\
0.750 & -126 & 7.0 & 405 & -220 & 22.1 & 2065 \\
0.831 & -81 & 7.2 & 507 & -19 & 17.6 & 1806 \\
0.926 & 135 & 13.1 & 853 & 300 & 10.1 & 1859 \\
\hline \hline
\end{tabular}

Notes to TABLE 5

Velocities are heliocentric in $\mathrm{km} \mathrm{s}^{-1}$.

Fluxes are in units of $10^{-15} \mathrm{erg} \mathrm{cm}^{-2} \mathrm{~s}^{-1}$.

eters and often incorporate polarimetric data (which we do not have) to constrain the geometry (e.g., Ferrario et al. 1989). However, at some orbital phases the lines parse nicely into base and peak components, so we used IRAF's SPLOT routine on the phase-binned spectra to "deblend" the line profiles. For each strong line we fit two Gaussians, one broad and one narrow. After guessing initial values for the centers of each component, we allowed the centers, widths, and strengths of the Gaussians to converge. Often the two components were very distinct and gave a remarkably good fit for such a simple model; in these cases the solutions among the three lines were found to be in good agreement, and not to be sensitive to the initial guess. In cases where the base and peak components appeared less distinct, the solutions were sensitive to the initial guess of line center. Table 5 shows the results of the fits to $\mathrm{H} \alpha$, which had the best signal to noise.

The behavior of the sharp component proved quite interesting. As can be seen from Fig. 12, this component is most distinct near phase 0.5. In $\mathrm{H} \alpha$ and $\mathrm{H} \beta$, the FWHM of the sharp component is nearly constant at about $380 \mathrm{~km} \mathrm{~s}^{-1}$ in

TABLE 6

Sharp Component Fits

\begin{tabular}{ccccc}
\hline \hline line & phase & amplitude & mean & $\sigma$ \\
\hline H $\alpha$ vel. & $-0.039 \pm 0.016$ & $171 \pm 15$ & $16 \pm 11$ & 27 \\
& $(0)$ & $167 \pm 17$ & $-6 \pm 13$ & 31 \\
H $\alpha$ flux & $0.252 \pm 0.017$ & $10.8 \pm 1.3$ & $6.3 \pm 0.9$ & 2.0 \\
& $(0.250)$ & $10.8 \pm 1.2$ & $6.3 \pm 0.8$ & 1.8 \\
H $\beta$ vel. & $-0.025 \pm 0.023$ & $150 \pm 19$ & $15 \pm 15$ & 33 \\
& $(0)$ & $148 \pm 17$ & $2 \pm 13$ & 31 \\
.H $\beta$ flux & $0.249 \pm 0.020$ & $13.8 \pm 2.0$ & $6.3 \pm 1.3$ & 3.1 \\
& $(0.250)$ & $13.8 \pm 1.8$ & $6.4 \pm 1.2$ & 2.8 \\
\hline \hline
\end{tabular}

Notes to TABLE 6

Fits are of form $x(t)=$ mean + amplitude $\sin [2 \pi(t-T) / P]$, where $x(t)$ is the velocity or flux and the period $P$ is flxed at $0.140245 \mathrm{~d}$. The phase given is that of $T$ with respect to HJD 2449255.64646. Flux units are $10^{-15} \mathrm{erg} \mathrm{cm}^{-2}$ $\mathrm{s}^{-1}$. 


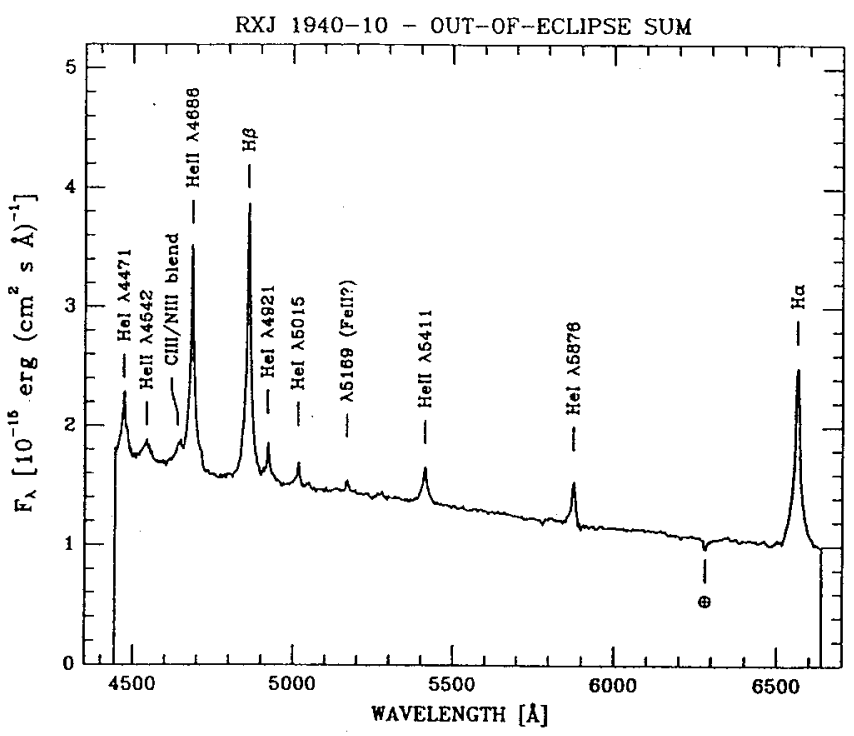

FiG. 14-Average out-of-eclipse spectrum.

the seven bins from phases 0.243 to 0.750 ; outside of this interval the FWHM increases, but the component becomes so indistinct that its physical significance is unclear. Table 6 gives parameters of sinusoidal fits to the sharp components in the 0.243-0.750 phase interval only. The fits show that over the phases in which the sharp component is welldefined, its velocity is fit nicely with a sinusoid at the phase expected for the red dwarf, crossing the mean near phase zero and reaching maximum a quarter-cycle later. Also, the sharp-component fluxes peak almost exactly at phase 0.5 , as expected if they originate in the heating of the secondary star's atmosphere by soft $X$ rays and EUV light from the vicinity of the white dwarf (since the secondary then presents its heated hemisphere to earthbound observers). Such heating effects and phase dependence are commonly observed in detached binaries consisting of red dwarfs and hot white dwarfs (e.g., Vennes and Thorstensen 1994).

\subsection{Average Spectrum}

Figure 14 shows the average spectrum well away from the eclipse, showing: bright emission lines of $\mathrm{H}$ and $\mathrm{He} \mathrm{I}$; strong He II lines at $\lambda 4686, \lambda 5411$, and $\lambda 4542$; and $\lambda 5169$ emission probably due to Fe II. This is pretty much "standard fare" for AM Her stars accreting at a high rate; the He II emission is almost certainly powered by photoionization by the abundant soft $\mathrm{X}$ rays produced from accretion.

\subsection{Comparison with Other Work, and Period Analysis}

The period we derive (12122 $\pm 9 \mathrm{~s})$ for the movement of the "whole line" agrees with the eclipse period, with the $12123 \pm 7$-s period derived by Watson et al. (1995) for the sharp component, and with the $12120 \pm 3$-s period derived by Staubert et al. (1994) for the whole line. Thus the radialvelocity measurements appear to be at least in rough agreement. Combining datasets, the epochs for blue-to-red crossing of the sharp component also agree: Watson et al. measure 9217.909 and we measure 9255.642 (average of $\mathrm{H} \alpha$ and $\mathrm{H} \beta$ ), yielding $12119 \pm 4 \mathrm{~s}$.
Staubert et al. measured the whole line and thus a blend of the sharp and broad components, so we cannot directly compare their results. But their measurements certainly suggest that the radial velocities are dominated by the behavior of the sharp line: their three epochs of measurement yield blue-tored crossings of $0.90,0.95$, and 0.01 on the eclipse ephemeris, compared to Watson et al.'s 0.90 and our 0.97 for the sharp line alone; and their semiamplitude is $180 \mathrm{~km} \mathrm{~s}^{-1}$, close to the two measured sharp-line semiamplitudes of $168 \pm 10 \mathrm{~km} \mathrm{~s}^{-1}$ (Watson et al. 1995) and $159 \pm 20 \mathrm{~km} \mathrm{~s}^{-1}$ (Table 6). Combining this data together, we estimate that during 1993 the blue-to-red crossing of the sharp component occurred at phase $0.95 \pm 0.03$ on the eclipse ephemeris.

Much less is known about the broad component. The phase relationship between broad and sharp components is fairly well specified in our data (Table 5); during that week, the $\mathrm{H} \alpha$ broad line led the sharp line by $0.136 \pm 0.025$ cycles. But a much more extensive study with the line components well separated will certainly need to be carried out, especially in view of the multiple periods present in this star.

\section{THE X-RAY PERIOD(S)}

Searches for periodic signals in the X-ray emission have a fascinating history. Tennant et al. (1981) first drew attention to sharp X-ray dips in the $H E A O \mathrm{~A}-2$ data, possibly recurring with a regular period. Beall et al. (1986) argued that the dips occurred as the satellite passed the geomagnetic equator, suggesting a terrestrial origin. But then Mittaz and BranduardiRaymont (1989) found a $12,100 \pm 100$-s period in a longuninterrupted EXOSAT ME (2-6 keV) observation, starting a trickle of theoretical papers to explain so strange an effect in an AGN. A reanalysis of the EXOSAT observation (Fiore et al. 1992) and new observations with the large-area counters on Ginga proved the periodicity beyond any doubt (Done et al. 1992; Leighly et al. 1993), turning the trickle into a flood.

Of course, the startling ROSAT discovery (Madejski et al. 1993; Staubert et al. 1994) that the periodic signal arises from a foreground cataclysmic variable changed everything. A phalanx of interested observers became suddenly uninterested; and a group of spectators, including ourselves, leaped into the breach. While no new $\mathrm{X}$-ray data have been reported since this discovery, we think that the major puzzles in the data are now basically solved, thanks to the long-baseline optical ephemerides and the ability to reinterpret the data in terms of magnetic CV. We try to demonstrate this below.

\subsection{Detailed Period History}

The original EXOSAT observation yielded a period of $12100 \pm 100 \mathrm{~s}$ (Mittaz and Branduardi-Raymont 1989; Fiore et al. 1992). Ginga observations lasted about 2 days, giving an accuracy of only about $50 \mathrm{~s}$; but using observations separated by 6 and 12 months, Done et al. (1992) found several acceptable precise, periods near 12,130 s. Done et al. favored $12132 \pm 3 \mathrm{~s}$, while King and Done (1993) cited $12144 \pm 5 \mathrm{~s}$. Madejski et al. (1993) showed convincing evidence for a 
TABLE 7

Timings of Dip, Events in Archival X-Ray Data

\begin{tabular}{|c|c|c|c|c|}
\hline $\begin{array}{l}(2440000+) \\
\text { HJD }\end{array}$ & Detector & Source & $\begin{array}{l}\text { Orbital } \\
\text { Phase }^{a}\end{array}$ & Remarks $^{b}$ \\
\hline 3626.5521 & HEAO A-1, A-2 & Beall et al. 1986 & .418 & \\
\hline 3626.6181 & $"$ & $"$ & .888 & \\
\hline 7644.7166 & Ginga LAC & Done et al. 1992 & .599 & 1 \\
\hline 7997.3935 & $"$ & Leighly et al. 1993 & .506 & 1 \\
\hline 7997.4556 & " & $"$ & .950 & 2 \\
\hline 7998.3767 & " & " & .518 & 1 \\
\hline 7998.4390 & " & " & .962 & 2 \\
\hline 7998.5154 & " & * & .507 & 1 \\
\hline 7998.5785 & " & " & .956 & 2 \\
\hline 8170.8758 & " & " & .594 & 3 \\
\hline 8171.0147 & " & $"$ & .584 & 3 \\
\hline 8915.0146 & ROSAT PSPC & Koenig 1994 & .983 & 4 \\
\hline 9079.0908 & " & Madejski et al. 1993 & .996 & 4 \\
\hline $\mathbf{9 0 7 7 . 8 9 8 0}$ & $"$ & " & .560 & 5 \\
\hline
\end{tabular}

Notes to TABLE 7

"On the 1993/4 eclipse ephemeris [Eq. (1)]. All phases are slightly later on the final ephemeris [Eq. (4)].

bRemarks:

1. Very sharp dip, very strong energy dependence; obvious sign of photoelectric absorption $\left(N_{\mathrm{H}} \sim 10^{24} \mathrm{~cm}^{-2}\right)$.

2. Slightly wider dips, slightly deeper.

3. Dips are very sharp and energy-dependent-probably of type 1 but incompletely resolved because of data gaps.

4. Narrow total (flat-bottomed) dip.

5. Wider, possibly somewhat irregular dip.

period of $12142 \pm 20 \mathrm{~s}$ for the 1993 April ROSAT observa-

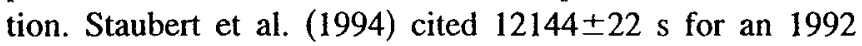
October ROSAT observation, and $12142 \pm 29 \mathrm{~s}$ from a reanalysis of the 1984 EXOSAT observation.

The period-finding enterprise in $X$ rays is riddled with problems. The coverage is sparse, and the distribution unfavorable since the candidate period is about twice the orbital period of satellites in near-Earth orbit. The wave form is complex and known to be highly variable. Some previous period estimates refer only to the narrow dips, some to the smooth variation (identified by Fourier-transform methods), and some to a combination ( $\chi^{2}$ test). Also, the X-ray dip signature is vastly less obvious than the optical eclipse; perusal of the literature shows that the $\mathrm{X}$-ray light curves containing certified narrow dips often contain other dips only slightly less prominent yet unremarked upon. Despite these complications, it is striking that all the best estimates for the $\mathrm{X}$-ray period (except for the original low-precision estimate) lie between the two periods known from optical photometry and spectroscopy to be the two fundamental clocks in the binary. We do not interpret this as evidence for yet another fundamental clock, but rather as a symptom of the mixing of two signals. We think that the fundamental period in hard $\mathrm{X}$ rays is very likely to be the precise 12150 -s period evident in the optical photometry, but this is convolved with a signal at $12116 \mathrm{~s}$.

That the latter is present, at least in the form of sharp dips, we will now show by studying the times of such events. A survey of the literature and X-ray data archives reveals 14 events since the original $H E A O-A$ discovery in 1978. These are presented in Table 7 with references and notes. In the fourth column of Table 7 we show the phase of the dips on the 1993-4 eclipse ephemeris. Basically there are two

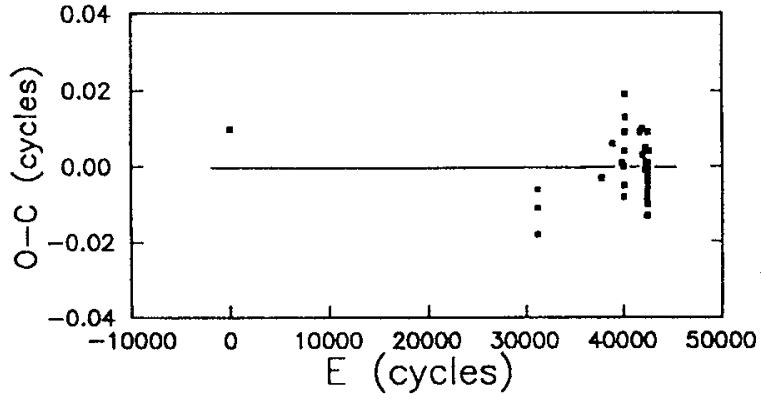

Fig. 15-O-C diagram, relative to Eq. (4), spanning the 16 years of eclipse timings.

groups tightly clustered in phase: a family near 0.55 and another near 0.96. Consider first the latter cluster. Its location near phase zero suggests that it may be the counterpart of the optical eclipse. Mean phases for these dips from the three satellites are: ROSAT, 0.989; Ginga, 0.956; HEAO-A, 0.888 . These departures from phase zero grow monotonically and nearly linearly as we look backward in time, a sign that the timings may obey a strict period slightly different from the test period. Adding these X-ray timings to the optical eclipse timings, we find that all data can be fit to a constant period, given by this ephemeris:

$$
\text { Minimum light }=3626.6167+0.1402349 E \text {. }
$$

The O-C diagram is shown in Fig. 15, indicating a satisfactory fit to all the timings. The period is very stable $\left(|\dot{P}|<10^{-10}\right)$.

\subsection{Hard X-ray Eclipse Waveform}

To further study the hypothesis that these X-ray eclipses are the counterparts to the optical eclipse, we folded the hard

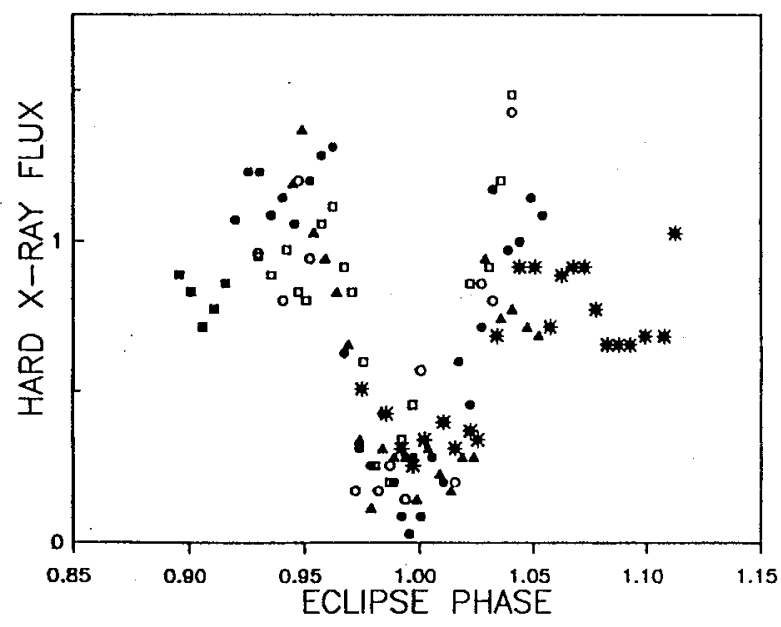

FIG. 16-Mean hard X-ray light curve in the vicinity of the eclipse, culled from published and archival data. Filled symbols indicate the four Ginga observations (Leighly et al. 1993), and open symbols indicate the HEAO-1 observations (Beall et al. 1986). The eclipse width and ingress/egress durations are consistent with optical data-confirming that they have a common origin, and strongly suggesting that the obscuration is by an opaque body (red star) rather than a diffuse gas cloud. 
X-ray light curves (near eclipse) on this ephemeris. We obtained coverage of three eclipses from Ginga (1-37 keV; Leighly et al. 1993), and one from HEAO-A (1-20 keV and 2-10 keV, from A-1 and A-2 respectively; Beall, Wood, and Yentis 1986). Figure 16 shows the composite waveform, with the out-of-eclipse flux levels from the two satellite observations set equal.

Measurement of Fig. 16 yields the following eclipse durations: ingress $=170 \mathrm{~s}$, egress $=80 \mathrm{~s}$, totality $=550 \mathrm{~s}$, and half-power eclipse $=680 \pm 40 \mathrm{~s}$. Because of erratic variability and uncertainties in background subtraction, the first three of these numbers are not highly trustworthy; but the eclipse width should be, and we note that it agrees satisfactorily with the optical estimate of $635 \pm 32 \mathrm{~s}$. We conclude that the reliable timing signatures of the eclipse (duration, and time of mid-eclipse) are the same in hard $X$ rays and optical light (4000-9000 A).

The actual depth of the eclipse in Fig. 16 is nominally a factor of $\sim 4-20$, depending on the background subtraction which is quite uncertain in these pointed observations with nonimaging detectors. The imaging observations of ROSAT (discussed previously by Madejski et al. 1993; Staubert et al. 1994; and Watson et al. 1995) are superior in this regard. These also show the X-ray eclipses (see especially Fig. 13 of Watson et al.), and from reanalysis of the two observations in the Heasarc Data Archive, we measure a half-power duration of $743 \pm 70 \mathrm{~s}$ for very soft $\mathrm{X}$ rays $(E<0.4 \mathrm{keV})$ and $702 \pm 50$ $\mathrm{s}$ for $0.4-2 \mathrm{keV} X$ rays. Thus there is weak prima facie evidence that the soft $\mathrm{X}$-ray eclipse is slightly wider than the optical/hard X-ray eclipse, but this needs study with more extensive coverage. The soft $X$-ray flux drops by a factor $>10$ (and probably $>20$ ) at mid-eclipse.

\section{WHAT ARE THESE PERIODS, ANYWAY?}

The simplest interpretation of the optical eclipse is that it represents the eclipse of the white dwarf by the red dwarf. Such deep and sharp eclipses are a characteristic feature of edge-on cataclysmic variables, whether magnetic or nonmagnetic, which lack bright accretion disks. The regularity, depth, and sharpness of the eclipse all point to this interpretation. Watson et al. (1995) argue instead that it represents the periodic occultation of the white dwarf by a portion of the mass-transfer stream, principally because the X-ray dip associated with the optical eclipse shows a pronounced energy dependence. We shall review the arguments here, and see why they greatly favor the simplest interpretation.

\subsection{Location of the Secondary Star}

There are four ways we could learn the location of the secondary in its orbit; we list them in rough order of decreasing reliability, although the appraisal of reliability really depends on the details of individual stars.

(1) The existence of deep, regular eclipses of the accreting star.

(2) An absorption-line radial-velocity curve of the secondary.
(3) A radial-velocity curve of narrow emission lines, assuming that the actual line profiles warrant a simple "broad +narrow" decomposition.

(4) Refiection effects off the secondary (restricted to orbital modulations of the narrow emission-line component; continuum variations are less useful since there are too many other potential sites for their origin).

(5) Infrared light curves; there is a double-humped wave form that is characteristic of a star filling its Roche lobe, and the times of maxima/minima can signify the orientation of the star. Now we consider how these clues might be used in the case of RX J19402-1025.

The first clue is a mighty one: the eclipses are indeed deep and regular, and occur at all energies studied to date. While there are some small irregularities in the eclipse profiles, we note that all well-studied eclipsing magnetic CVs show some tendency for auxiliary dips to occur preceding first contact or following last contact (V471 Tau: Jensen et al. 1986; DP Leo: Robinson and Cordova 1994; UZ For: Osborne et al. 1988; Bailey et al. 1993). We interpret this to mean merely that the vicinity of the secondary star is cluttered with gas streams, which can be fairly dense in an edge-on binary. In particular, there is a general expectation of seeing such effects just prior to first contact, because at that phase the gas stream just beginning to fall towards the white dwarf (which is probably the narrowest and hence densest part of the stream) is close to the line of sight. There are now four other eclipsing magnetic CVs known, and we anticipate that close study will reveal such effects in most of them.

The second clue is not yet available. Spectral features of the secondary are only readily visible at mid-eclipse (Watson et al. 1995), preventing the needed observation around the full orbit.

The third clue is available and discussed in Sec. 6. The profiles looked fairly simple at those times when the narrow lines are strong. The lines move on the 12116-s period, and their source reaches blue-to-red crossing ("inferior conjunction," when interpreted as arising from dynamical motions) at phase $0.95 \pm 0.03$.

The fourth clue is also available. The fluxes of the narrow lines (Tables 5 and 6) are strongly modulated at $12116 \mathrm{~s}$, reaching maximum at orbital phase 0.5 and disappearing below measurement limits in and near eclipse. They thus display the expected phase dependence of a "heated secondary" seen at high binary inclination.

The fifth clue is unavailable, because the secondary is too faint. AM Her stars are famous for their intense cyclotron fluxes in the infrared, so it may be difficult to find a wavelength at which the secondary dominates.

Thus the three clues which can be used all rule in favor of the view that the secondary is at inferior conjunction at phase zero. But the narrow lines reach blue-to-red crossing at phase 0.95; why not zero exactly? Well, this seems to be the normal pattern for AM Her stars. There are five other stars in which the location of the secondary is known securely and precisely from other evidence (clues 1 and/or 2), and where a narrow component can bey distinctly followed in the undulating emission line. These are listed in Table 8 with the relevant numbers and references. In all cases the narrow component 
TABLE 8

Do the Narrow Emission Lines Track the True Motion of the Secondary?

\begin{tabular}{|c|c|c|}
\hline Star & $\begin{array}{l}\text { Phase by which lines } \\
\text { lead secondary star }\end{array}$ & Source \\
\hline DP Leo & $0.05(2)$ & Biermann et al. 1985 \\
\hline ST LMi & $0.06(3)$ & Mukai \& Charles 1987, Bailey et al. 1985 \\
\hline QQ Vul & 0.13 & Mukai \& Charles 1987 \\
\hline AM Her & $0.02(1)$ & $\begin{array}{l}\text { Young \& Schneider 1979; Young, Schneider, } \\
\text { \& Schectman 198I }\end{array}$ \\
\hline $\begin{array}{l}\text { UZ For } \\
\text { (low state) }\end{array}$ & 0.00 & Beuermann et al. 1988 \\
\hline $\begin{array}{l}\text { UZ For } \\
\text { (high state) }\end{array}$ & $0.13(3)$ & Ferrario et al. 1989, Allen et al. 1989 \\
\hline BY Cam & $0.10(2)$ & Silber et al. 1992 \\
\hline DQ Her & $0.00(5)$ & Martell, Horne, Gomer, \& Price 1994 \\
\hline $\mathrm{RX} \mathrm{J19402}$ & $0.05(2)$ & This paper \\
\hline IX Vel* & $-0.02(3)$ & Beuermann \& Thomas 1990 \\
\hline $\begin{array}{l}\text { SS Cyg* } \\
\text { (outburst) }\end{array}$ & $-0.02(2)$ & Hessman 1986 \\
\hline $\mathrm{UX} \mathrm{UMa}^{*}$ & $-0.08(5)$ & Schlcgel, Honeycutt, \& Kaitchuck 1983 \\
\hline RW Tri* & $\sim 0.00(6)$ & Kailchuck, Honcycutt, \& Schlegel 1983 \\
\hline $\begin{array}{l}\text { DW UMa* } \\
\text { (low state) }\end{array}$ & $0.01(2)$ & Dhillon, Jones, \& Marsh 1994 \\
\hline $\begin{array}{l}\text { IP Peg* } \\
\text { (post-outburst) }\end{array}$ & $-0.02(2)$ & Hessman 1989 \\
\hline
\end{tabular}

Notes to TABLE 8

Note 1. To be eligible for this list, a star must show another clue to the secondary's location, of greater reliability and precision. Usually this means a deep eclipse, an absorption-line radial-velocity curve, or an infrared light curve showing "ellipsoidal" variations.

Note 2. Asterisk means non-magnetic, or probably non-magnetic. Should still roughly apply, if the relevant physics is photoionization in the stream leaving the $L_{1}$ point. The main difference is probably that an accretion disk may shield the stream from ionizing radiation.

Note 3. Answer to query in table heading: Almost but not quite. For the magnetics, they lead the secondary by a small amount; the shift averages $\sim 0.06$ but probably rises in states of higher accretion. For the non-magnetics, the average shift is -0.02 (2) and so is basically consistent with an origin purely in the secondary.

"leads" the true motion of the secondary by a small amount, averaging $\sim 0.06$ in binary phase. As to what might be causing this shift, consider the other slight anomaly in the lines: we estimate from the data in Fig. 13 that the emission-line eclipse is symmetrical about phase $0.014 \pm 0.003$. Why not zero exactly? Well, consider the gas recently departed from the secondary. To conserve angular momentum it must curve forward in its orbit, meaning that it is maximally eclipsed slightly after the true dynamical conjunction of the stars. Further, by virtue of falling a bit towards the white dwarf, this gas will have acquired a little redshift-so at true conjunction the observed velocity will have already passed its blue-to-red crossing, producing the sense of the phase shifts seen among the magnetic systems of Table $8 .^{7}$

\footnotetext{
${ }^{7}$ We also include information concerning nonmagnetic systems, which sometimes show narrow emission lines very closely in phase with the secondary. Typically, these components appear during or just after an episode
}

Thus, we think that the narrow emission line consists of a main component which arises from the heated secondary, and a smaller component arising from low-velocity gas which has just started to fall. The latter dominates only near eclipse phases, when the stronger part is completely hidden. The broad component arises farther downstream in the flow, after the infall velocities greatly exceed the orbital velocities. If $P_{\text {rot }} \neq P_{\text {orb }}$, as is very likely in this star, there is an additional complication that the broad component could follow either period (or both!) depending on whether the gas flow is yet fully channeled by the white-dwarf's magnetic field.

\subsection{The Other Period: Rotation}

Observations during 1994 revealed variable circular polarization at the few percent level, the hallmark of a magnetic CV (Buckley 1994). Most of the energy should be released quite close to the accreting star, and since the flow is roughly channeled along the white-dwarf's field lines, the accretion structures near the white dwarf are expected to wheel about on the white-dwarf's rotation period. Thus we really expect that period, not the orbital period, to dominate the continuum radiation at all wavelengths, as well as the polarized flux. These predictions appear to be satisfied. There is a strong 12150 -s optical component, stable in period, and both hard $\mathrm{X}$-rays and circular polarization are periodic with periods consistent with this (respectively, 12144 \pm 5 s, Koenig 1994; and the first harmonic of $12140 \pm 12 \mathrm{~s}$, Buckley 1994). It appears that all signs of accretion structures near the white dwarf are consistent with a 12150 - (or possibly 12148-) $\mathrm{s}$ period.

\subsection{The Slow Variation at $12,116 \mathrm{~s}$}

The observed slow variation at $12,116 \mathrm{~s}$ does not fit particularly well into the above scheme. During our two years of coverage, it was manifest as a rather good sinusoid peaking at eclipse phase $0.449(13)$ and $0.454(18)$. It is possible that this effect arises from the reprocessing of $X$ rays in the atmosphere of the secondary. The secondary subtends a solid angle of $\sim 3 \%$ of $4 \pi$ sr at the white dwarf, so reprocessing produces a flux $\sim 0.03 F_{\text {acc }}$, where $F_{\text {acc }}$ is the flux from accretion light. The observed signal at $12,116 \mathrm{~s}$ shows a flux of $\sim 5 \times 10^{-12} \mathrm{erg} / \mathrm{cm}^{2} / \mathrm{s}$, and the observed hard X-ray flux $F_{\mathrm{HX}}(1-50 \mathrm{keV})$ is $1.5 \times 10^{-11} \mathrm{erg} / \mathrm{cm}^{2} / \mathrm{s}$ (Beall et al. 1986). Thus hard $\mathrm{X}$ rays alone appear to be insufficient to power the signal. But the energetics could still be feasible, if other components of accretion light (soft $\mathrm{X}$ rays, largely hidden by interstellar absorption, and cyclotron radiation) exceed hard $\mathrm{X}$ rays by a factor of $\sim 10$. This degree of excess is rare, but not unprecedented, in AM Her stars (King and Watson 1987; Ramsay et al. 1994).

The observed $\Delta \phi=-0.05$ phase shift is another problem. In view of this problem, we should consider another solution: that the slow variation does exactly signify the location

of high accretion, when a strongly exciting source of UV photons might be guessed (or in a few cases known) to be present. The data appear to be essentially consistent with a strict origin in the secondary, suggesting that the accretion disk may shield the mass-transfer stream from the ionizing photons. 
of the secondary, which would locate it at inferior conjunction at eclipse phase 0.95 . Does this contradict other evidence? Well, the sharp component reaches blue-to-red crossing at eclipse phase 0.95 , which obviously is in excellent agreement. There remains the major eclipse event-is anything poised to eclipse the white-dwarf 0.05 cycles after true dynamical conjunction? We cannot think of anything, and generally expect structures on the orbit-following side to be more dispersed and sheared (and thus poorly equipped to produce narrow, deep, and stable eclipses).

These issues make us nervous but not enough to make us "abandon ship." More accurate estimates of the poorly observed components of accretion light are needed to assess the likelihood of a heating effect.

\subsection{The Other X-ray Dip}

In Sec. 7 we saw that dips in archival X-ray data seem to cluster around two eclipse phases. One appears to occur exactly on a schedule, and we interpret it as an eclipse by the secondary star. The other occurs around eclipse phase 0.58 , but wanders by $\sim 0.04$ in both directions. From the very similar phases of the three Ginga dips observed on days $7997 / 8$, it seems likely that the wanderings are quite slow compared to the orbital time scale. Nine events in Table 7 occur near this phase: those labeled with remarks 1,3 , and 5 , plus the first of the HEAO-A dips.

What causes these dips? The observations show a very sharp energy dependence. If the hard X-ray spectra are modeled with a constant temperature and photoelectric absorption in cold gas, then the absorbing column density $N_{\mathrm{H}}$ rises from $10^{22}$ to $10^{25} \mathrm{~cm}^{-2}$ in the dips (see Fig. 8 of Leighly et al. 1993). This is an extremely plausible model, because absorption is the only known process which can produce so sharp an energy dependence, and the absorbing cloud must be cool $\left(<10^{8} \mathrm{~K}\right)$ because the short duration of the dips indicates that it must be located far from the white dwarf.

Similar dips, obviously due to absorption in a cool cloud, are commonly observed in synchronous AM Her systems (see Watson et al. 1987 for an excellent review). They are generally interpreted as due to absorption in the accretion column. But such effects are expected to occur on the rotation period, contrary to observation in RX J19402-1025. Examination of the phases of the nine timings on the 50-day supercycle does not show any preferred phase-suggesting that the dips really do occur on the orbital period (not merely at times when the two clocks were accidentally in phase). However, the latter conclusion is not firm and should be more severely tested with additional X-ray data and a longterm supercycle ephemeris.

The question remains: why should there be such a cloud, and why at phase 0.58 ? Since observations seem to require that the cloud follow the orbit rather than the rotation, we assume that it represents a structure associated with the mass-transfer stream. But the normal "bright spot" at the outer edge of a putative accretion disk would be expected to transit across the line of sight to the white dwarf at about $\Phi_{\text {orb }}=0.80-0.85$, in very poor agreement with observation. Therefore we expect that the absorption occurs farther down- stream, where the falling gas starts to loop around the white dwarf.

\section{ASYNCHRONISM IN AM HERS}

An asynchronous AM Her star is a pretty strange beast, technically violating the strict definition of the class which requires synchronism. Two other examples are known: BY Camelopardalis $(=\mathrm{H} 0538+608$; Remillard et al. 1986; Mason et al. 1991; Silber et al. 1992) and V1500 Cygni (=Nova Cygni 1975; Stockman et al. 1988; Schmidt et al. 1994). In the spirit of aggressive stellar nomenclature, we should probably call this small group "the BY Cam stars." Nevertheless, it should be stressed that all three really appear to be bona fide AM Hers; their properties do not exactly typify the "average" AM Her star, but their resemblance is sufficiently thorough to persuade us completely of their membership. To put it another way, their deviation from the norm is no greater than the intrinsic dispersion in the obvious members of the class.

So how did they come to be asynchronous? We reject the idea that they are just now, for the first time, approaching or leaving synchronism. V1500 Cyg is observed to be approaching synchronism on a time scale of $170 \mathrm{yr}$ (Schmidt et al. 1994), and the AM Her lifetime is probably in the range $1-5 \times 10^{9}$ yr. From these numbers we estimate only one chance in $\sim 10^{7}$ of accidentally catching a given star in its first approach or last departure from synchronism, and this cannot be reconciled with the observed statistics of 3/54. It is much more likely that AM Hers have episodic departures from synchronism, and spend $-5 \%$ of their lives being mildly asynchronous.

As to the causes of the departures, the classical nova outburst is a tempting choice. All AM Her stars should have such outbursts, since they contain degenerate dwarfs accreting hydrogen at a low rate. One of the three stars, V1500 Cyg, is known to have had a recent outburst, and has shown a slowly changing period (now approaching synchronism) ever since. The nova recurrence time for these stars of moderately low $\dot{M}$ is probably in the range $(2-20) \times 10^{4} \mathrm{yr}$, so a synchronization time like the $170 \mathrm{yr}$ observed in V1500 Cyg implies that such stars spend $0.1 \%-1.0 \%$ of their lives being asynchronous.

This is in mild disagreement with observation. The reason may lie in the fact that V1500 Cyg is a very recent nova and hence might show an initial relocking time scale slightly different from its long-term average. The speed class of the V1500 Cyg nova eruption is also extreme (one of the fastest in history), suggestive of a massive white dwarf and therefore a small moment of inertia. A few more years of observation of all three stars is likely to clarify this question substantially.

The orbital periods of all three stars are the same to within $1 \%$. We ascribe no special significance to this, except to say that the BY Cam class should tend to be preferentially populated by long-period AM Hers. This is because the strength of the magnetic lock should be much weaker for long-period systems, since the 'dipole falls off rapidly with distance. A simple calculation (Patterson 1994) suggests that the mag- 
netic lock scales like $B M^{-3.1} P^{-2.8}$, where $B$ is the white dwarf's magnetic field, $M$ is the white dwarf's mass, and $P$ is the binary period. Thus a $3.4 \mathrm{hr}$ binary is $\sim 7 \times$ less tightly gripped than a typical AM Her star with $P=1.7 \mathrm{hr}$, other factors being equal. This would be exaggerated further if two other plausible and popular ideas about $\mathrm{CV}$ evolution are correct: that the evolutionary arrow is from long to short $P$, and that white-dwarf masses are progressively eroded with ongoing classical nova outbursts. The $M^{-3.1}$ factor would then work to weaken the magnetic lock in long-period systems.

On the basis of this theory (which we actually believe!), we expect that most asynchronous AM Hers should be in long-period systems. ${ }^{8}$

\section{SUMMARY AND THE VIEW AHEAD}

(1) We report on two seasons of photometry, which demonstrate the existence of deep eclipses at 12,116 s, and smooth variations at both 12,116 and $12,150 \mathrm{~s}$. The eclipse period is stable but phase shifts of up to $\sim 60 \mathrm{~s}$ seem to occur. The longer period appears to be stable over one and possibly two seasons, but evaluation of long-term stability requires additional photometry. It is very likely that the eclipse period is the underlying orbital period of the binary, while the white dwarf rotates with $P=12150 \mathrm{~s}$.

(2) The two signals beat together with a supercycle of 50 d, so the light curve returns to the same appearance on this period.

(3) Close inspection of the data reveals a possible alias for the longer period of $12147.8 \mathrm{~s}$, corresponding to a supercycle of 54.1 days. Further photometry should be planned to distinguish between these possibilities.

(4) Spectroscopy reveals a fairly clean decomposition into sharp and broad components. The sharp component obviously follows the 12116-s orbital period. It arises from reprocessing of (predominantly soft) $\mathrm{X}$ rays in or near the atmosphere of the secondary, and hence largely tracks the motion of the secondary. Some contribution to the sharp component also probably arises from gas streams leaving the secondary. The broad component presumably arises from infall motion farther downstream, but observations are still too sparse to specify its behavior in detail.

(5) We review the history of period studies from X-ray observations, in light of the precise ephemerides derived here. It seems likely that the true period in hard $X$ rays is $12150 \mathrm{~s}$, arising from the white-dwarf's rotation. But there are also sharp dips occurring at phase 0.0 and 0.6 on the $12116 \mathrm{~s}$ ephemeris. The former seems to be a counterpart of the optical eclipse, arising from occultation by the secondary. The sharp energy-dependent dip at phase 0.6 surely arises from the transit of a gas cloud across the line of sight, but we are not able to place any natural candidate at the "scene of

\footnotetext{
${ }^{8}$ And since we do more or less believe it, we should remark that it very significantly affects the details of the " $5 \%$ " argument just presented. It would illuminate this problem greatly to repeat the calculation with explicit and detailed estimates for the dependence of nova recurrence time on $M$, $\dot{M}, P$, and $B$.
}

the crime." This is presumably the same dip seen in optical light at this phase.

(6) The slow variation at $12116 \mathrm{~s}$ suggests an origin in reprocessing of accretion light by the secondary. But there are problems with energetics, and it is somewhat puzzling that the observed phase is advanced by 0.05 . We discuss this issue with no convincing resolution.

(7) This star joins BY Cam and V1500 Cyg as the third known asynchronous AM Her star, in a total population of 54. A rough calculation is consistent with the simple hypothesis that these are normal AM Hers with their synchronism temporarily disrupted by a nova outburst. We expect that most of these BY Cam stars will be found at relatively long orbital period.

(8) For a long time we have been waiting for a bright eclipsing AM Her star, to access the great diagnostic information supplied in principle when the secondary knifes across the luminous structures near the white dwarf. This is such a star, and it comes with a bonus feature. Because the rotation is not quite synchronous, we can study the eclipse waveform at many rotational phases-ranging from "pole on" to "edge on" to "pole away." The eclipses are Nature's own little CAT scan of the accretion column. Emission-line, polarimetric, and multicolor photometric studies across eclipse for a variety of phases in the 50-day cycle are likely to yield a vast retum of information, which we eagerly await.

The above story is a long one, and it pays to have many friends. We would like to thank Brad Schaefer, Fred Ringwald, Anouk Shambrook, and Jonathan Kemp for their contributions to the data described in this paper; Carolyn Weinberg for superb assistance with data analysis; and Michael Koenig, Jules Halpern, Karen Leighly, Carole Haswell, and Mike Watson for helpful discussions. The research was supported in part by NASA Grant No. NAGW-2565 and NSF Grant No. AST93-12367.

\section{REFERENCES}

Allen, R. G., Berriman, G., Smith, P. S., and Schmidt, G. D. 1989 , ApJ, 347, 426

Bailey, J., Wickramasinghe, D. T., Ferrario, L., Hough, J. H., and Cropper, M. 1993, MNRAS, 261, L31

Beall, J. H., Wood, K. S., and Yentis, D. J. 1986, ApJ, 308, 563

Beuermann, K. 1993, private communication

Beuermann, K., et al. 1988, A\&A, 195, L15

Beuermann, K., Stella, L, and Patterson, J. 1987, ApJ, 316, 360

Beuermann, K., and Thomas, H.-C. 1990, A\&A, 230, 326

Biermann, P., et al. 1985, ApJ, 293, 303

Buckley, D. A. 1994, private communication

Cropper, M. 1990, Space Sci. Rev., 54, 195

Dhillon, V. S., Jones, D. H. P., and Marsh, T. R. 1994, MNRAS, 266,859

Done, C., Madejski, G. M., Mushotzky, R. F., Turner, T. J., Koyama, K., and Kuneida, H. 1992, ApJ, 400, 138

Ferrario, L., Wickramasinghe, D. T., Bailey, J., Tuohy, I. R., and Hough, J. H. 1989, ApJ, 337, 832

Ferrario, L., Wickramasinghe, D. T., and Tuohy, I. R. 1989, ApJ, 341,327

Fiore, F., Massaro, E., and Bárone, P. 1992, A\&A, 261, 405

Hessman, F. V. 1986, ApJ, 300, 794 
Hessman, F. V. 1989, AJ, 98, 675

Jensen et al. 1986

Kaitchuck, R. H., Honeycutt, R. K., and Schlegel, E. M. 1983, ApJ, 267,239

King, A. R., and Done, C. 1993, MNRAS, 264, 388

King, A. R., and Watson, M. G. 1987, MNRAS, 277, 205

Koenig, M. 1994, private communication

Leighly, K., Kuneida, H., Tsusaka, Y., Awaki, H., and Tsuruta, S 1993, preprint

Madejski, G., et al. 1993, Nature, 365, 626

Mason, P. A., Liebert, J., and Schmidt, G. D. 1991, ApJ, 346, 951

Mittaz, J. P. D., and Branduardi-Raymont, G. 1989, MNRAS, 238, 1029

Mukai, K., and Charles, P. A. 1987, MNRAS, 226, 209

Osborne, J. P., et al. 1988, ApJ, 328, L45

Patterson, J. 1984, ApJS, 54, 443

Patterson, J. 1994, PASP, 106, 209

Patterson, J., Halpern, J. P., and Skillman, D. R. 1993, IAU Circ., No. 5852

Ramsay, G., et al. 1994, MNRAS, 270, 692

Remillard, R. A., et al. 1986, ApJ, 302, L11

Roberts, D. H., Lehar, J., and Dreher, J. W. 1987, AJ, 93, 968

Robinson, C. R., and Cordova, F. A. 1994, in Interacting Binaries, ed. A. W. Shafter, ASP Conf. Ser. 56, p. 146
Schlegel, E. M., Honeycutt, R. K., and Kaitchuck, R. H. 1983, ApJS, 53, 397

Schmidt, G. D., Stockman, H. S., and Lieben, J. E. 1994, ApJ, in press

Schneider, D. P., and Young, P. J. 1980, ApJ, 238, 946

Silber, A., Bradt, H. V., Ishida, M., Ohashi, T., and Remillard, R. A. 1992, ApJ, 389, 704

Skillman, D. R. 1993, Sky \& Telescope, 85, 5:83

Skillman, D. R., and Patterson, J. 1993, ApJ, 417, 298

Staubert, R., Koenig, M., Friedrich, S., Lamer, G., Sood, R. K., James, S. D., and Sharma, D. P. 1994, A\&A, 288, 51

Stockman, H. S., Schmidt, G. D., and Lamb, D. Q. 1988, ApJ, 332, 282

Tennant, A. F, and Mushotzky, R. F. 1983, ApJ, 264, 92

Tennant, A. F., Mushotzky, R. F., Boldt, E. A., and Swank, J. H. 1981, ApJ, 251, 15

Vennes, S., and Thorstensen, J. 1994, AJ, submitted

Watson, M. G., King, A. R., and Williams. G. A. 1987, MNRAS, 226,867

Watson, M. G., et al. 1995, MNRAS, in press

Young, P. J., and Schneider, D. P. 1979, ApJ, 230, 502

Young, P. J., Schneider, D. P., and Shectman, S. A. 1981, ApJ, 245, 1043 